\title{
BINOMIAL ENUMERATION ON DISSECTS
}

\author{
BY \\ MICHAEL HENLE
}

\begin{abstract}
The Mullin-Rota theory of binomial enumeration is generalized to an abstract context and applied to rook polynomials, order invariants of posets, Tutte invariants of combinatorial geometries, cycle indices and symmetric functions.
\end{abstract}

1. Introduction. In [10] Mullin and Rota discuss polynomial sequences $\left\{\mathbf{p}_{n}\right\}$ of binomial type, that is satisfying the identity

$$
\mathbf{p}_{n}(x+y)=\sum_{k}\left(\begin{array}{l}
n \\
k
\end{array}\right) \mathrm{p}_{k}(x) \mathrm{p}_{n-k}(y)
$$

for all nonnegative integers $n, x$, and $y$. Given such a sequence, Mullin and Rota define a family of differential operators on the vector space of polynomials, prove a series of expansion and isomorphism theorems, and apply these to the proof of identities and the solution of combinatorial problems. Identities like (1) abound in combinatorics. For example, rook polynomials, symmetric polynomials and the cycle indices of permutation groups all satisfy similar identities. In this paper we generalize the ideas of Mullin and Rota to apply to these other identities. Many of the results generalize easily, the proofs being modeled on those of [10] or the paper of Garsia [5]. Applications include many classical results. Rather than provide new results on specific problems, the object of this paper is to show that the idea of binomial enumeration unifies a large portion of enumerative combinatorics.

The principal tool is the category of dissects first introduced in [7]. §2 of . the present paper gives the necessary background on dissects. The definitions given in this section differ from those in [7] in some small ways. $\S \S 3,4$, and 5 contain the theory of binomial enumeration together with applications. We have reserved the theory of symmetric functions for separate treatment in $\S \S 6,7$, and 8 .

We wish to stress the debt this paper owes to that of Mullin and Rota and to the later paper of Rota [14]. Our contribution is in showing how widely applicable their ideas are.

Received by the editors February 14, 1973.

AMS (MOS) subject classifications (1970). Primary 05A15, 05A19; Secondary 05 B35, 06A10.

Key words and phrases. Incidence algebra, symmetric functions. 


\section{Dissects.}

DEFINITION 1. Let $\mathbf{S}$ be a set whose elements $x$ may be taken apart or dissected according to some principle or other. We symbolize this by $x \rightarrow a \oplus b$, indicating $x$ is dissected into the elements $a$ and $b$ of $\mathbf{S}$ in that order. Each such dissection is given a multiplicity or incidence coefficient $m\left[\begin{array}{c}x \\ a\end{array}\right]$ from the real numbers. We set $m\left[\begin{array}{c}x \\ a b\end{array}\right]=0$ in case $x \nrightarrow a \oplus b$ so that $m$ is defined on the whole cartesian product $\mathbf{S} \times \mathbf{S} \times \mathbf{S}$. The pair $(\mathbf{S}, m)$ is called a dissect if (a) $m\left[\begin{array}{l}x \\ a\end{array}\right]$ for given $x$ is zero for all but a finite number of pairs $(a, b)$ and given $a$ and $b$ is zero for all but a finite number of $x$, and (b) for all $x, a, b$, $c \in \mathbf{S}$,

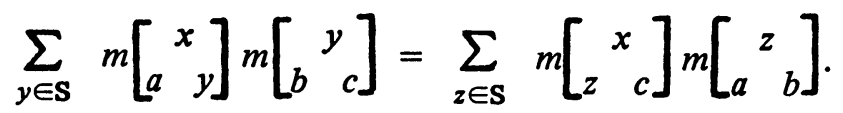

The quantity given in (A) is called the multinomial incidence coefficient of the

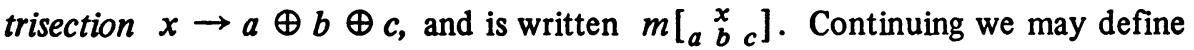
$m\left[\begin{array}{llll} & x & \\ a & b & c & d\end{array}\right]$ and so forth. It frequently occurs that the incidence coefficients take only the values zero and one. They are then completely determined by the dissections $x \rightarrow a \oplus b$. We refer to these as the usual incidence coefficients on $\mathbf{S}$. A dissect $(\mathbf{S}, m)$ has an identity $e$ if $m\left[\begin{array}{l}x \\ x\end{array}\right]=m\left[\begin{array}{l}e^{x} \\ x\end{array}\right]=1$ for all $x \in \mathbf{S}$, but all other incidence coefficients involving $e$ are zero.

EXAMPLES OF DISSECTS. (1) The set $Z$ of nonnegative integers is a dissect with dissections $x \rightarrow k \oplus x-k$ for $0 \leqslant k \leqslant x$. The usual incidence coefficients are then $s\left[\begin{array}{c}x \\ a b\end{array}\right]=\delta_{x, a+b}$. We can also choose the incidence coeffi-

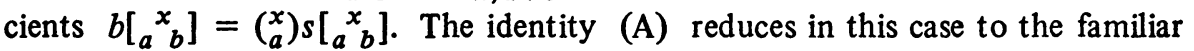
identity $\left(\begin{array}{l}x \\ n\end{array}\right)\left(\begin{array}{l}n \\ k\end{array}\right)=\left(\begin{array}{l}x \\ k\end{array}\right)\left(\begin{array}{l}x-k \\ n-k\end{array}\right)$. The multinomial incidence coefficients, $b\left[\begin{array}{lll}a & x & c\end{array}\right]$, and so forth, are the usual multinomial coefficients. Zero is an identity for $\mathbf{Z}$ with either $s$ or $b$ as incidence coefficients.

The incidence coefficients $e\left[\begin{array}{c}x \\ a\end{array}\right]=b\left[\begin{array}{cc}x-a \\ x-b a+b-x\end{array}\right]$ are quite different. Here $e\left[\begin{array}{l}x \\ a\end{array}\right]$ counts the number of ways of choosing subsets of cardinalities $a$ and $b$ from a set of $x$ elements so as to exhaust the set. In this case we have $x \rightarrow a \oplus b$ whenever $a, b \leqslant x \leqslant a+b$. The multinomial incidence coefficient $e\left[\begin{array}{lll}a & x & \\ a & b\end{array}\right]$ counts the number of ways of choosing three subsets of cardinalities $a, b$ and $c$ in order to exhaust a set of cardinality $x$. The identity (A) says this number can be obtained by first choosing sets of cardinalities $a$ and $y$ to exhaust the set of $x$ elements, and then choosing subsets of $b$ and $c$ elements to exhaust the set of $y$ elements; or by first choosing sets of $z$ and $c$ elements to exhaust the set of $x$ elements, and then choosing subsets of $a$ and $b$ elements to exhaust the set of $z$ elements. The dissect $(Z, e)$ has no identity.

(2) Chessboards. Let $B$ be the set of all finite subsets of a doubly infinite chessboard. For $B, B_{1}, B_{2}$ in $B$ set $B \rightarrow B_{1} \oplus B_{2}$ when $B$ is the union 
of $B_{1}$ and $B_{2}$ and $B_{1}$ and $B_{2}$ have no elements common to a single row or column. Then $\mathbf{B}$ is a dissect with the usual incidence coefficients. The empty chessboard acts as an identity.

(3) Posets. Let $\mathbf{P}$ be the set of all finite partially ordered sets where we identify isomorphic partially ordered sets. Let $s$ [respectively $s^{\prime}$ ] denote the usual incidence coefficients when we define $P \rightarrow P_{1} \oplus P_{2}$ for $P, P_{1}, P_{2}$ in $\mathrm{P}$ to mean $P=P_{1}+P_{2}$, the direct sum of $P_{1}$ and $P_{2}$ [respectively $P=P_{1} / P_{2}$, the ordinal sum of $P_{1}$ and $P_{2}$ ]. The direct sum is defined by taking the union of $P_{1}$ and $P_{2}$ and no relations between the two sets; the ordinal sum is also defined by taking the union but setting $p_{1}>p_{2}$ for all $p_{1} \in P_{1}$ and $p_{2} \in P_{2}$. Both $(\mathbf{P}, s)$ and $\left(\mathbf{P}, s^{\prime}\right)$ are dissects for which the empty poset acts as an identity.

(4) Combinatorial geometry. Let $\mathbf{C}$ be the dissect of all combinatorial pregeometries with the usual incidence coefficients defined by setting $G \rightarrow G_{1}$ $\oplus G_{2}$ whenever $G$ is the direct sum of $G_{1}$ and $G_{2}$. We refer the interested reader to [2] for a summary of the theory of combinatorial geometry.

(5) Permutation groups. Let $\mathbf{G}$ be the dissect of all groups of permutations acting on finite sets. For $G \in \mathbf{G}$, we will write $S_{G}$ for the set upon which $G$ acts. We identify groups which are isomorphic as permutation groups; that is, for which the sets on which the groups act can be put into one-to-one correspondence inducing an isomorphism of the groups. Define $G \rightarrow G_{1} \oplus G_{2}$ whenever $G$ is the usual direct sum of $G_{1}$ and $G_{2}$ in the sense of permutation groups; that is, the group $G$ is the direct sum of the groups $G_{1}$ and $G_{2}$ acting in the obvious way on the disjoint union of $S_{G_{1}}$ and $S_{G_{2}}$. An identity is supplied by allowing $\mathbf{G}$ to contain the trivial group acting on the empty set.

(6) Any set $\mathbf{S}$ becomes a dissect upon defining the trivial incidence coefficients $p\left[\begin{array}{ll}x \\ a & b\end{array}\right]$ to be one, when $x=a=b$, and zero otherwise.

Definition 2. Let $(\mathbf{S}, m)$ be a dissect. Define $I^{*}(\mathbf{S}, m)[I(\mathbf{S}, m)]$ as the set of all real-valued functions on $\mathbf{S}$ [which are zero at all but a finite number of points of $\mathbf{S}$ ]. Both $I^{*}(\mathbf{S}, m)$ and $I(\mathbf{S}, m)$ are algebras with convolution multiplication defined by

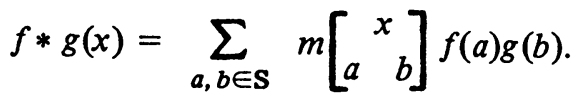

These are the incidence algebras of the dissect $\mathbf{S}$ (see [7] for the connection with the classical incidence algebras of [18]). When no confusion can result we omit explicit mention of the incidence coefficients. At times this is not possible. For example $I^{*}(\mathrm{Z}, s)$ is isomorphic to the algebra of formal power series, while $I^{*}(\mathrm{Z}, b)$ is isomorphic to the algebra of exponential generating functions. Note that $I^{*}(\mathrm{~S}, p)$, where $p$ are trivial incidence coefficients, is the algebra of pointwise multiplication of functions on $\mathbf{S}$. 
Definition 3. Let $(\mathrm{S}, m),(\mathrm{T}, n)$ be dissects. A morphism [ ${ }^{*}$ morphism] from $(S, m)$ to $(T, n)$ is a real-valued function $p$ defined on $S \times T$ and satisfying (a) for all $x, y \in \mathrm{S}, a \in \mathrm{T}$.

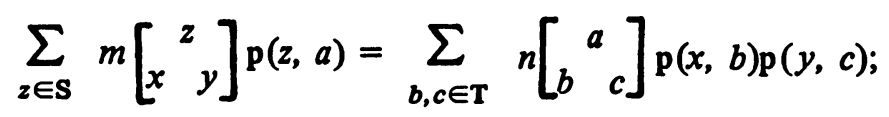

(b) for $a \in \mathrm{T}, \mathrm{p}(x, a)$ is zero for all but a finite number of $x \in \mathrm{S}$. [(b*) for $x \in \mathrm{S}, \mathrm{p}(x, a)$ is zero for all but a finite number of $a \in \mathrm{T}$.] Let $(\mathrm{V}, r)$ be a third dissect, and $q:(T, n) \rightarrow(V, r)$ a second morphism [ ${ }^{*}$ morphism]. The composition of $\mathbf{p}$ and $\mathbf{q}$ is defined by

$$
\left(\mathrm{q}^{\circ} \mathrm{p}\right)(x, u)=\sum_{a \in \mathrm{T}} \mathrm{p}(x, a) \mathrm{q}(a, u), \quad x \in \mathrm{S}, u \in \mathrm{V} .
$$

The reader who is unhappy that morphisms in the category of dissects are not functions from $\mathbf{S}$ to $\mathbf{T}$ should consult [7] where an account is given of the genesis of these definitions, and should verify that composition is well defined, that $q \circ p$ is a morphism [ ${ }^{*}$ morphism] from $(S, m)$ to $(V, r)$ and that either notion (morphism or ${ }^{*}$ morphism) makes dissects a category. The identity in either case on $(S, m)$ is the Kronecker delta, $\delta(x, y)$, which is one if $x=y$, zero otherwise.

Given a morphism $\left[{ }^{*}\right.$ morphism] $\mathbf{p}(\mathbf{S}, m) \rightarrow(\mathbf{T}, n)$, we define an algebra morphism $\mathrm{p}: I(\mathrm{~T}, n) \rightarrow I(\mathrm{~S}, m)\left[\mathrm{p}: I^{*}(\mathrm{~T}, n) \rightarrow I^{*}(\mathrm{~S}, m)\right]$ by setting

$$
\mathrm{p} f(x)=\sum_{a \in \mathrm{T}} \mathrm{p}(x, a) f(a), \quad x \in \mathrm{S},
$$

for $f \in I(\mathrm{~T}, n)\left[I^{*}(\mathrm{~T}, n)\right]$. No confusion results from using the same letter for the dissect and algebra morphisms. We also use the notation $\mathbf{p}_{a}$ for the particular function $\mathrm{p}_{a}(x)=\mathrm{p}(x, a)$ in $I(\mathbf{S})\left[I^{*}(\mathbf{S})\right]$. This definition makes $I(\mathbf{S})$ $\left[I^{*}(\mathbf{S})\right]$ a contravariant functor. When we speak of the range of $\mathrm{p}$ we always mean the range of the induced algebra morphism. Also when we refer to $\mathbf{p}$ as a monomorphism we mean the algebra morphism. In this case the elements $\left\{\mathbf{p}_{a}\right.$, $a \in \mathbf{T}\}$ form a basis for the range of $\mathbf{p}$. In a sense we are simply studying the algebra $I(\mathbf{S})$ or $I^{*}(\mathbf{S})$ with a distinguished basis. More poetically, we regard our subject as the interplay between combinatorics and algebra that results when a distinguished basis is used.

The identity (B) is our generalization of (1.1). In most applications, however, neither condition (b) nor $\left(b^{*}\right)$ of Definition 3 is satisfied, so we require the following more general notion.

Definition 4. A morph $\mathbf{p}$ from the dissect $(\mathrm{S}, m)$ to the dissect $(\mathrm{T}, n)$ is a real-valued function $\mathbf{p}$ defined on $\mathbf{S} \times \mathbf{T}$ and satisfying condition (a) of 
Definition 3. We cannot define composition for morphs, but the induced map p exists as an algebra morphism from $I(\mathrm{~T})$ to $I^{*}(\mathrm{~S})$.

EXAMPLES. (1) Counting functions and compositions. A polynomial sequence of binomial type is a sequence $\left\{\mathrm{p}_{n}\right\}$ of polynomials which defines a morph from $(\mathrm{Z}, s)$ to $(\mathrm{Z}, b)$, and for which $\mathrm{p}_{n}$ is of degree $n$ exactly. These sequences, as explained in [10], tend to count classes of functions from a set of $n$ elements to a set of $x$ elements. The sequence $\mathrm{d}_{n}=x^{n}$ counts the class of all such functions. For further examples one should consult [10].

Morphs from $(Z, s)$ to $(Z, s)$ are also of interest. They tend to count compositions instead of functions. Thus $\mathrm{a}(x, n)=\left(\begin{array}{c}x+n-1 \\ n\end{array}\right)$ counts all compositions of $n$ into $x$ parts, and $b(x, n)=\left(\begin{array}{l}x \\ n\end{array}\right)$ counts compositions using only zeros and ones. The identity (B) takes the form

$$
\mathrm{b}(x+y, n)=\sum_{k=0}^{n} \mathrm{~b}(x, k) \mathrm{b}(y, n-k)
$$

reflecting the obvious way compositions into $x+y$ parts decompose into compositions using $x$ parts and $y$ parts. $\mathbf{b}$ is a ${ }^{*}$ morphism, in fact a *automorphism. $\mathbf{b}^{-1}(x, n)=(-1)^{x+n}\left(\begin{array}{l}x \\ n\end{array}\right)$. This inversion of $\mathbf{b}$ is an expression of the well-known orthogonality relation

$$
\sum_{n \geq 0}(-1)^{x+n}\left(\begin{array}{l}
x \\
n
\end{array}\right)\left(\begin{array}{l}
n \\
k
\end{array}\right)=\delta_{x, k} .
$$

Morphisms are easily found by counting compositions from which zero parts are excluded. For example $\mathrm{c}(x, n)=\left(\begin{array}{c}n-1 \\ x-1\end{array}\right)$ counts all such compositions and $f(x, n)=\left(\begin{array}{c}x \\ n-x\end{array}\right)$ counts compositions using only ones and twos.

We obtain morphs from $(\mathrm{Z}, s)$ to $(\mathrm{Z}, e)$ by counting classes of functions from a set $X$ of $x$ elements onto a set $N$ of $n$ elements. Let $G(X, N)$ stand for the class of all such functions, and set $g(x, n)=|G(X, N)|$. Letting $X \oplus Y$ stand for the disjoint union of $X$ and $Y$, we note that every $f \in$ $G(X \oplus Y, N)$ when restricted to $X$ is onto a unique subset $H \subseteq N$, and similarly $f$ restricted to $Y$ is onto a set $K \subseteq N$ so that $H \cup K=N$. Conversely any pair of functions, one from $G(X, H)$ and one from $G(Y, K)$, may be pieced together to form a function in $G(X \oplus Y, N)$. Symbolically

$$
G(X \oplus Y, N)=\sum_{H, K \subseteq N ; H \cup K=N} G(X, H) \otimes G(Y, K),
$$

where $\otimes$ stands for the operation of piecing functions together. Passing to cardinalities,

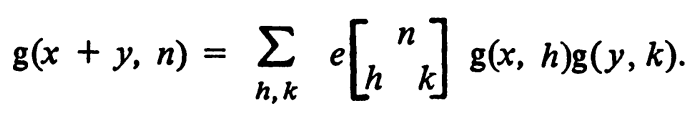


(2) Rook polynomials. For $B \in \mathrm{B}, n \in \mathrm{Z}$, we define $\mathrm{r}(B, n)$ as the number of ways of placing $n$ rooks on the board $B$ without any two rooks attacking each other. These are the numbers used as coefficients in the classical rook polynomials (see $[13$, p. 165]). They satisfy the identity

$$
\mathrm{r}\left(B_{1} \oplus B_{2}, n\right)=\sum_{k=0}^{n} \mathrm{r}\left(B_{1}, k\right) \mathrm{r}\left(B_{2}, n-k\right) .
$$

In other words $\mathbf{r}$ is a ${ }^{*}$ morphism from $(\mathbf{B}, s)$ to $(\mathbf{Z}, s)$.

(3) Order polynomials. There exists a great profusion of morphs defined on P. For example, the number of chains in a poset and the number of antichains each defines a morph from $\mathbf{P}$ to $(Z, s)$; the first with respect to the incidence coefficients $s^{\prime}$ on $\mathbf{P}$, the second with respect to the incidence coefficients $s$. We collect these and similar examples into a single morph by defining $\mathrm{j}(P, Q)$ for $P$, $Q \in \mathbf{P}$ to be the number of ways the poset $Q$ may be embedded in the poset $P$. Then one easily verifies the identities

$$
\begin{aligned}
\mathrm{j}\left(P_{1}+P_{2}, Q\right) & =\sum_{Q \rightarrow Q_{1}+Q_{2}} \mathrm{j}\left(P_{1}, Q_{1}\right) \mathrm{j}\left(P_{2}, Q_{2}\right), \\
\mathrm{j}\left(P_{1} / P_{2}, Q\right) & =\sum_{Q \rightarrow Q_{1} / Q_{2}} \mathrm{j}\left(P_{1}, Q_{1}\right) \mathrm{j}\left(P_{2}, Q_{2}\right) .
\end{aligned}
$$

In other words $\mathbf{j}$ is a ${ }^{*}$ morphism $(\mathbf{P}, s) \rightarrow(\mathbf{P}, s)$ and $\left(\mathbf{P}, s^{\prime}\right) \rightarrow\left(\mathbf{P}, s^{\prime}\right)$.

In a somewhat different direction, we define the ${ }^{*}$ morphism $\mathrm{e}(P, n)$ as the number of strict order preserving maps of $P$ onto a chain of $n$ elements. The numbers $\mathrm{e}(P, n)$ were used by Stanley [15] in defining the order polynomial of a partially ordered set. One easily verifies the identity

$$
\mathrm{e}\left(P_{1} / P_{2}, n\right)=\sum_{k=0}^{n} \mathrm{e}\left(P_{1}, k\right) \mathrm{e}\left(P_{2}, n-k\right)
$$

so that $\mathbf{e}$ is a ${ }^{*}$ morphism from $\left(\mathbf{P}, s^{\prime}\right)$ to $(\mathbf{Z}, s)$.

(4) Subgeometry generating function. Let $\left(\mathrm{Z}^{2}, s\right)$ be the dissect of ordered pairs of nonnegative integers with usual incidence coefficients arising from the dissections $(m, n) \rightarrow(h, k) \oplus(m-h, n-k)$. For $G \in \mathbf{C}$ we define $s(G, m, n)$ as the number of subgeometries of $G$ with corank $m$ and nullity $n$ obtaining a *morphism from $(C, s)$ to $\left(Z^{2}, s\right)$. In other words $s$ satisfies the identity

$$
\mathrm{s}\left(G_{1} \oplus G_{2}, m, n\right)=\sum_{h, k=0}^{m, n} \mathrm{~s}\left(G_{1}, h, k\right) \mathrm{s}\left(G_{2}, m-h, n-k\right) .
$$

The numbers $\mathrm{s}(G, m, n)$ are the coefficients of the subgeometry generating function of $G$. A proof of (7) is found in [2, p. 250]. 
(5) Cycle indices. Let $\mathrm{Pn}$ be the dissect of all partitions of the nonnegative integers. Let $\lambda=\left(\lambda_{1}, \lambda_{2}, \cdots, \lambda_{r}\right)=\left(1^{r_{1}}, 2^{r_{2}}, \cdots\right)$ be a partition. For future reference we collect here a number of special notations concerning $\lambda$ :

$$
\begin{gathered}
\|\lambda\|=\lambda_{1}+\lambda_{2}+\cdots=r_{1}+2 r_{2}+\cdots, \quad|\lambda|=r=r_{1}+r_{2}+\cdots, \\
\lambda !=r_{1} ! r_{2} ! \cdots, \quad \Pi(\lambda)=\lambda_{1} \lambda_{2} \cdots, \\
\operatorname{sgn}(\lambda)=(-1)^{\|\lambda\|+|\lambda|, \quad b(\lambda)=|\lambda| ! / \lambda ! .}
\end{gathered}
$$

The last quantity, $b(\lambda)$, is the number of permutations of the parts of $\lambda$. Now define $\lambda \rightarrow \lambda_{1} \oplus \lambda_{2}$ when $\lambda$ is the union of the parts of $\lambda_{1}$ and $\lambda_{2}$ (as a multiset). The dissections $\lambda \rightarrow \lambda_{1} \oplus \lambda_{2}$ were termed separations by MacMahon [9]. Allowing the number zero to have one partition, this partition becomes an identity for Pn.

For $\lambda \in \mathrm{Pn}, G \in \mathrm{G}$, we define $\mathrm{p}(G, \lambda)$ as the number of permutations of $G$ of cycle type $\lambda$ divided by the order of $G$. These numbers are the coefficients of the cycle index of $G$. They satisfy the identity

$$
\mathrm{p}\left(G_{1} \oplus G_{2}, \lambda\right)=\sum_{\lambda \rightarrow \lambda_{1} \oplus \lambda_{2}} \mathrm{p}\left(G_{1}, \lambda_{1}\right) \mathrm{p}\left(G_{2}, \lambda_{2}\right),
$$

so that $\mathbf{p}$ is a *morphism from (G, $s)$ to $(\mathbf{P n}, s)$, where $s$ are the usual incidence coefficients on Pn. There are other sets of incidence coefficients on $\mathbf{G}$ and $\mathbf{P n}$ of interest with respect to $\mathbf{p}$. Let $p$ be the trivial incidence coefficients on Pn with the one difference that $p\left[{ }_{\lambda}^{\lambda} \lambda\right]=\lambda ! \pi(\lambda)$. On $G$ we set $G \rightarrow G_{1} \oplus G_{2}$ whenever $G_{1}$ and $G_{2}$ act on the same set and there exist permutations $\sigma_{1} \sigma_{2}$ on that set such that $G=$ $\left(\sigma_{1} G_{1} \sigma_{1}^{-1}\right) \cap\left(\sigma_{2} G_{2} \sigma_{2}^{-1}\right)$. Recall that, since we identify isomorphic permutation groups, two groups $G_{1}$ and $G_{2}$ may be considered to act on the same set if they act on sets of the same cardinality. Define incidence coefficients $t$ on $\mathbf{G}$ so that $t\left[G_{1}{ }_{1}^{G} G_{2}\right]$ counts the number of pairs of permutations $\sigma_{1}$ and $\sigma_{2}$ described above. Then $\mathrm{p}$ is also a *morphism from $(\mathrm{G}, t)$ to $(\mathrm{Pn}, p)$ in that $\mathrm{p}$ satisfies

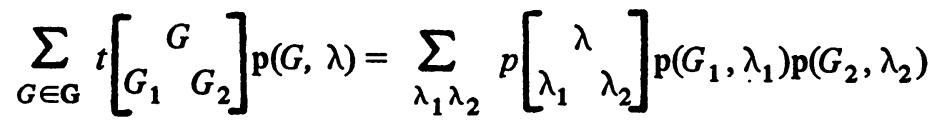

$$
\begin{aligned}
& =\lambda ! \pi(\lambda) \mathrm{p}\left(G_{1}, \lambda\right) \mathrm{p}\left(G_{2}, \lambda\right) .
\end{aligned}
$$

This is the Redfield-Master theorem. A proof may be found in [12], where it is also shown that in applications (9) is equivalent to the Pólya enumeration theorem.

\section{Operators and expansions.}

Definition 5. Let $(S, m)$ be a dissect. For each $x \in \mathrm{S}$ define the shift operator $E_{x}$ on $I^{*}(\mathbf{S})$ by 


$$
E_{x} f(y)=\sum_{z \in \mathbf{S}} m\left[\begin{array}{cc}
z \\
x & y
\end{array}\right] f(z), \quad f \in I^{*}(\mathbf{S})
$$

These operators play a fundamental role in the following theory. They satisfy the multiplication rule

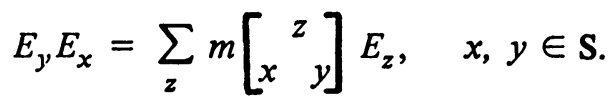

The proof is as follows,

$$
\begin{aligned}
E_{y} E_{x} f(w) & =\sum_{z} m\left[\begin{array}{ll}
z \\
y & w
\end{array}\right] E_{x} f(z)=\sum_{z} \sum_{t} m\left[\begin{array}{cc}
z \\
y & w
\end{array}\right] m\left[\begin{array}{cc}
t \\
x & z
\end{array}\right] f(t) \\
& =\sum_{t} f(t) \sum_{z} m\left[\begin{array}{cc}
t \\
x & z
\end{array}\right] m\left[\begin{array}{cc}
z \\
y & w
\end{array}\right]=\sum_{t} f(t) \sum_{z} m\left[\begin{array}{ll}
t \\
z & w
\end{array}\right] m\left[\begin{array}{cc}
z \\
x & y
\end{array}\right] \\
& =\sum_{z} m\left[\begin{array}{ll}
z \\
x & y
\end{array}\right] \sum_{t} m\left[\begin{array}{ll}
t \\
z & w
\end{array}\right] f(t)=\sum_{z} m\left[\begin{array}{ll}
z \\
x & y
\end{array}\right] E_{z} f(w) .
\end{aligned}
$$

As this sort of computation occurs rather often in what follows, we will save much ums and space by abbreviating. We shall let the letter labels of the various identities stand for invocation of the identity itself and asterisks stand for interchanges of summation. Thus the preceding computation would be abbreviated $(S)(S)^{*}(A)^{*}(S)$.

Let $\mathrm{p:}(\mathrm{S}, m) \rightarrow(\mathrm{T}, n)$ be a morph. For the remainder of the paper we shall assume (S, $m$ ) has an identity and $\mathbf{p}$ is a monomorphism. We shall omit the (usually trivial) verification of these properties in examples.

Definition 6. Let $P$ be the range of $p$. For each $c \in T$ define the differential operator $P_{c}$ on $P$ by setting

$$
P_{c} \mathrm{p}_{a}=\sum_{b \in \mathrm{T}} n\left[\begin{array}{c}
a \\
b
\end{array}\right] \mathrm{p}_{b}, \quad a \in \mathrm{T}
$$

and extending by linearity from the basis $\left\{\mathbf{p}_{a} \mid a \in T\right\}$ to all of $P$. The differential operators satisfy the multiplication rule,

$$
P_{c} P_{d}=\sum_{b} n\left[\begin{array}{cc}
b \\
c & d
\end{array}\right] P_{b}, \quad c, d \in \mathrm{T},
$$

as one verifies using $(D)(D)^{*}(A)^{*}(D)$.

Rewriting using (S), (B) becomes

$$
E_{x} \mathbf{p}_{a}(y)=\sum_{b, c \in \mathbf{T}} n\left[\begin{array}{c}
a \\
b
\end{array}\right] \mathbf{p}_{b}(x) \mathbf{p}_{c}(y)
$$

for $x, y \in \mathrm{S}, a \in \mathrm{T}$. Further, by using (D) and linearity, we obtain for $f \in P$ 


$$
E_{x} f=\sum_{c \in \mathrm{T}} P_{c} f(x) \mathrm{p}_{c} .
$$

Thus (B) becomes a connection between the shift and differential operators. This shows that $P$ is an invariant subspace for the shift operators. Next we verify the crucial property of the differential operators: shift invariance, that is $P_{c} E_{x}=E_{x} P_{c}$ for all $x \in \mathrm{S}, c \in \mathrm{T}$. To prove this it suffices to apply the lefthand side to a typical element $\mathbf{p}_{a}$ of our basis of $P$ and follow the computation $(\mathrm{E})(\mathrm{D})^{*}(\mathrm{~A})^{*}(\mathrm{E})(\mathrm{D})$.

Let $P^{*}$ denote the set of all shift invariant operators on $P$. We introduce an inner product connecting $P^{*}$ and $P$ by setting

$$
\langle Q, f\rangle=Q f(e), \quad Q \in P^{*}, f \in P,
$$

where $e$ is the identity of $\mathbf{S}$. This actually makes sense for any element $f \in$ $I^{*}(\mathbf{S})$ and operator $Q$ so long as $Q f$ is defined. In particular we have

$$
\left\langle E_{x}, f\right\rangle=f(x), \quad f \in I^{*}(\mathbf{S}) .
$$

The usefulness of the differential operators will now appear.

FIRST EXPANSION THEOREM. Every element $f$ of $P$ has an expansion $f=\Sigma_{a \in \mathrm{T}}\left\langle P_{a}, f\right\rangle \mathbf{p}_{a}$.

Proof. Set $x=e$ in (F).

COROLlaRy. $\left\langle P_{a}, \mathrm{p}_{b}\right\rangle=\delta_{a, b}$.

SECOND EXPANSION THEOREM. Every element $Q$ of $P^{*}$ has an expansion

$$
Q=\sum_{a \in \mathrm{T}}\left\langle Q, \mathrm{p}_{a}\right\rangle P_{a} .
$$

Proof. We first note that the right-hand side of the above equation does define an element of $P^{*}$ since each $f \in P$ is a linear combination of finitely many elements $p_{b}$, and each $p_{b}$ is annihilated by all but a finite number of the operators $P_{a}$. Thus in the application of the right side of (1) to $f$ only a finite number of terms are not zero. Let $Q^{\prime}$ denote this operator. Clearly $Q^{\prime} \in P^{*}$. Now for $b \in \mathbf{T}$,

$$
\left\langle Q^{\prime}, \mathrm{p}_{b}\right\rangle=\sum_{a \in \mathrm{T}}\left\langle Q, \mathrm{p}_{a}\right\rangle\left\langle P_{a}, \mathrm{p}_{b}\right\rangle=\left\langle Q, \mathrm{p}_{b}\right\rangle
$$

By linearity $\left\langle Q^{\prime}, f\right\rangle=\langle Q, f\rangle$ for all $f \in P$. Thus, following Garsia [5], the proof is completed by the following lemma.

LEmma. If $Q, Q^{\prime}$ are shift invariant operators such that $\langle Q, f\rangle=\left\langle Q^{\prime}, f\right\rangle$ for all $f \in P$, then $Q=Q^{\prime}$. 
Proof. For any $x \in \mathrm{S}$,

$Q^{\prime} f(x)=\left\langle E_{x}, Q^{\prime} f\right\rangle=\left\langle E_{x} Q^{\prime}, f\right\rangle=\left\langle Q^{\prime}, E_{x} f\right\rangle=\left\langle Q, E_{x} f\right\rangle=\left\langle E_{x}, Q f\right\rangle=Q f(x)$.

From (M) we deduce the following

ISOMORPHISM THEOREM. The algebra $P^{*}$ of shift invariant operators is isomorphic to $I^{*}(\mathrm{~T}, n)$.

From these results it follows easily that $P^{*}$ is the dual space, via our inner product, of $P$, and that $\left\{\mathbf{p}_{b} \mid b \in \mathbf{T}\right\}$ and $\left\{P_{a} \mid a \in \mathbf{T}\right\}$ are dual bases. We mean dual bases only in the sense that $\left\langle P_{a}, \mathbf{p}_{b}\right\rangle=\delta_{a, b}$, since the operators $\left\{P_{a} \mid a \in T\right\}$ do not form a basis in the usual sense for $P^{*}$. A partial converse is given in the following

DUAL BASIS THEOREM. Let $\left\{\mathrm{q}_{b} \mid b \in \mathrm{T}\right\}$ be a basis for $P$, and let $\left\{Q_{a} \mid\right.$ $a \in \mathrm{T}\}$ be a dual basis in the above sense for $P^{*}$. Supposing the operators $Q_{a}$ satisfy the multiplication rule $(\mathrm{M})$, the $\mathrm{q}_{b}$ define a morph from $(\mathrm{S}, m)$ to $(\mathrm{T}, n)$ whose corresponding differential operators are the $Q_{a}$.

Proof. It follows from the hypothesis that each $f \in P$ has an expansion $f=\Sigma_{a}\left\langle Q_{a}, f\right\rangle \mathbf{q}_{a}$. We next prove successively that

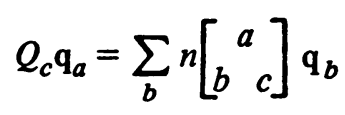

and

$$
E_{x} \mathbf{q}_{a}=\sum_{b, c} n\left[\begin{array}{ll}
a \\
b & c
\end{array}\right] \mathrm{q}_{b}(x) \mathrm{q}_{c}
$$

by expanding the left-hand sides, since

$$
\left\langle Q_{b}, Q_{c} \mathbf{q}_{a}\right\rangle=\left\langle Q_{b} Q_{c}, \mathbf{q}_{a}\right\rangle=n\left[\begin{array}{cc}
a \\
b & c
\end{array}\right]
$$

using the identity (M), and

$$
\left\langle Q_{c}, E_{x} \mathbf{q}_{a}\right\rangle=\left\langle Q_{c} E_{x}, \mathbf{q}_{a}\right\rangle=\left\langle E_{x}, Q_{c} \mathbf{q}_{a}\right\rangle=Q_{c} \mathbf{q}_{a}(x)=\sum_{b} n\left[\begin{array}{cc}
a \\
b & c
\end{array}\right] \mathbf{q}_{b}(x)
$$

using (2). This concludes the proof since (3) is equivalent to the defining identity (B) of morphs (see also (F)), and (2) shows that $\left\{Q_{c}\right\}$ is the corresponding family of differential operators.

EXAMPLES. (1) Polynomial sequences of binomial type $\left\{\mathbf{p}_{n}\right\}$. The restriction on the degrees of the polynomials $\mathbf{p}_{n}$ ensures that these sequences define monomorphs. The differential operators are defined by 


$$
P_{k} \mathbf{p}_{n}=\left(\begin{array}{l}
n \\
k
\end{array}\right) \mathbf{p}_{n-k}
$$

and satisfy the product rule $P_{k} P_{m}=\left(\begin{array}{c}k+m \\ k\end{array}\right) P_{k+m}$, from which it follows that all the operators $P_{k}$ are generated from $P=P_{1}$ by the relation

$$
P_{k}=P^{k} / k ! \text {. }
$$

In the case $\mathrm{d}(x, n)=x^{n}, D$ is the usual derivative operator, and the first expansion theorem gives the usual Taylor expansion. The reader is referred to [10] and [14] for more examples of these sequences and many expansions relating to them.

The question arises, given a shift invariant operator $P$, whether there exists a sequence of binomial type whose differential operators are determined by $\boldsymbol{P}$ as in (5). Mullin and Rota found the following characterization of these operators. A delta operator $P$ is a shift invariant operator for which $P 1$ is zero and $P x$ is a nonzero constant.

THEOREM (MULLIN-RotA). An operator $P$ arises from a polynomial sequence of binomial type $\left\{\mathbf{p}_{n}\right\}$ by setting $P_{\mathbf{p}_{n}}=n \mathbf{p}_{n-1}$ if and only if $P$ is $a$ delta operator.

Proof. We shall only sketch the proof, since the details are not significantly different from the proof given in [10]. Given an operator $P$ arising from a polynomial sequence as described above, the binomial identity (1.1) allows one to conclude that $\mathrm{p}_{0}=1$ and $\mathrm{p}_{k}(0)=0$ for $k>1$. It follows immediately that $P$ is a delta operator. Conversely given a delta operator $P$ one first proves $[10$, p. 181] that $P$ reduces degrees. One may then construct a polynomial sequence by setting $\mathrm{p}_{0} \equiv 1$ and, proceeding inductively, solving the "differential equation" $P \mathrm{p}_{n}=n \mathrm{p}_{n-1}$ with the initial condition $\mathrm{p}_{n}(0)=0, n>0$. One thereby obtains a basis for the polynomials for which the set of operators $\left\{P_{k}\right\}$ defined by (5) are a dual basis. By the dual basis theorem the sequence $\left\{\mathbf{p}_{n}\right\}$ is of binomialtype, and the operators $\left\{P_{k}\right\}$ are the corresponding differential operators.

(2) Permutations with restricted position. Consider the morph $\mathbf{r}$ from $(\mathrm{B}, s)$ to $(\mathrm{Z}, s)$. The differential operators $R_{k}$ are all powers of the operator $R=R_{1}$. To find a closed form for $R$, for each $B \in \mathrm{B}$ let $B \oplus s$ denote the board formed from $B$ by adding a square not in any row or column presently occupied by a square of $B$. From (2.3), $\mathrm{r}(B \oplus s, n)=\mathrm{r}(B, n)+\mathrm{r}(B, n-1)$, or $R \mathbf{r}_{n}(B)=\mathbf{r}_{n-1}(B)=\mathbf{r}_{n}(B \oplus s)-\mathbf{r}_{n}(B)$. It follows by linearity that $R f(B)=$ $f(B \oplus s)-f(B)$ for all $f \in R$, where $R$ is the range of r. In order to use the expansion theorems we must characterize the functions of $R$. The following argument and its parallels in succeeding examples was suggested by the work of Brylawski [2]. Let $B \in \mathbf{B}$ and let $x$ be a square of $B$ which is not the only square of $B$ in its row and/or column. Let $B-x$ denote $B$ with the square $x$ removed, 
and let $B / x$ denote $B$ with all the squares of $x$ 's row and column removed. As is well known,

$$
\mathbf{r}_{n}(B)=\mathbf{r}_{n}(B-x)+\mathbf{r}_{n-1}(B / x)=\mathbf{r}_{n}(B-x)+\mathbf{r}_{n}(B / x \oplus s)-\mathbf{r}_{n}(B / x) .
$$

By linearity every $f \in R$ must satisfy the identity

$$
f(B)=f(B-x)+f(B / x \oplus s)-f(B / x) .
$$

Conversely we claim $R$ contains all functions satisfying the identities of this type. Let $f$ be such a function. We form the expansion $\Sigma_{n \in Z}\left\langle R^{n}, f\right\rangle \mathbf{r}_{n}$ according to the first expansion theorem. Let $f^{\prime}$ denote the sum of this series which exists even if an infinite number of coefficients are nonzero since $\mathbf{r}$ is a ${ }^{*}$ morphism. The question is whether $f^{\prime}=f$. Both $f$ and $f^{\prime}$ satisfy (6) which expresses their values at $B$ in terms of their values on boards with fewer cells, provided of course that $B$ has a cell $x$ as described above. By induction $f$ and $f^{\prime}$ will be equal if we prove they are equal for the boards with no such cells, that is for the boards $s^{n}=s \oplus s \oplus \cdots \oplus s$ ( $n$ times). We find that

$$
R^{n} f(B)=\sum_{k}(-1)^{n+k}\left(\begin{array}{l}
n \\
k
\end{array}\right) f\left(B \oplus s^{k}\right),
$$

so that

$$
\left\langle R^{n}, f\right\rangle=\sum_{k}(-1)^{n+k}\left(\begin{array}{l}
n \\
k
\end{array}\right) f\left(s^{k}\right) .
$$

Finally using the orthogonality relation (2.2)

$$
\begin{aligned}
f^{\prime}\left(s^{m}\right) & =\sum_{n} \sum_{k}(-1)^{n+k}\left(\begin{array}{l}
n \\
k
\end{array}\right) f\left(s^{k}\right) \mathbf{r}_{n}\left(s^{m}\right) \\
& =\sum_{k} \sum_{n}(-1)^{n+k}\left(\begin{array}{l}
n \\
k
\end{array}\right)\left(\begin{array}{l}
m \\
n
\end{array}\right) f\left(s^{k}\right)=f\left(s^{m}\right) .
\end{aligned}
$$

We are now free to expand any function satisfying the identities (6) in terms of r. For example consider the boards $B$ contained in a given $M \times M$ square; these form a subdissect of $B$ to which our preceding argument applies word for word. Let $f_{h}(B)$ be the number of ways of placing $M$ rooks on the square so that exactly $h$ fall on $B$. $f_{h}$ satisfies (6). We find by inclusion-exclusion that

$$
f_{h}\left(s^{k}\right)=\sum_{i}(-1)^{i+h}\left(\begin{array}{l}
k \\
i
\end{array}\right)\left(\begin{array}{l}
i \\
h
\end{array}\right)(M-i) !
$$

Then 


$$
\begin{aligned}
f_{h}(B) & =\sum_{n}\left\langle R^{n}, f_{h}\right\rangle \mathbf{r}_{n}(B)=\sum_{n} \mathbf{r}_{n}(B) \sum_{k}(-1)^{n+k}\left(\begin{array}{l}
n \\
k
\end{array}\right) f_{h}\left(s^{k}\right) \\
& =\sum_{n} \mathbf{r}_{n}(B) \sum_{k}(-1)^{n+k}\left(\begin{array}{l}
n \\
k
\end{array}\right) \sum_{i}(-1)^{i+h}\left(\begin{array}{l}
k \\
i
\end{array}\right)\left(\begin{array}{l}
i \\
h
\end{array}\right)(M-i) ! \\
& =\sum_{n}(-1)^{n+h}\left(\begin{array}{l}
n \\
h
\end{array}\right)(M-n) ! \mathbf{r}_{n}(B),
\end{aligned}
$$

a well-known result.

(3) A-invariants. The morph $e:\left(\mathrm{P}, s^{\prime}\right) \rightarrow(\mathrm{Z}, s)$ has a theory parallel to the preceding example. The differential operators $E_{n}$ are given by $E_{n} f(P)=$ $f(P / \mathrm{n})$, where $\mathrm{n}$ is a chain of length $n$. Since $E_{n}$ is a shift, no confusion results from this dual use of the letter $E$. To describe the range $E$ of e, let $P$ be a partially ordered set, and $x, y$ a pair of unrelated elements of $P$. Following Stanley [17], let $P_{x}^{y}$ denote the partially ordered set $P$ with the relation $x<y$ added and all relations implied thereby, and let $P_{x y}$ denote the partially ordered set $P$ with $x$ and $y$ identified. Then one verifies easily that the elements $f \in E$ satisfy the identity

$$
f(P)=f\left(P_{x}^{y}\right)+f\left(P_{y}^{x}\right)+f\left(P_{x y}\right) .
$$

Functions satisfying (7) are called $A$-invariants. Examples of $A$-invariants are the order polynomial $(-1)^{|P|} \Omega_{n}(P)$, where $\Omega_{n}(P)$ is the number of strict maps from $P$ into an $n$ chain, and $(-1)^{|P|} L(P)$, where $L(P)$ is the number of order ideals of $P$. Expanding these in terms of the functions $e_{n}$, we obtain various results of Stanley [17].

(4) Tutte invariants. The differential operators of the subgeometry generating function morph s: $(\mathrm{C}, s) \rightarrow\left(\mathrm{Z}^{2}, s\right)$ are generated by the two operators $S_{1}=$ $S_{1,0}$ and $S_{2}=S_{0,1}$ and the relation $S_{i, j}=S_{1}^{i} S_{2}^{j}$. One easily verifies that

$$
S_{1} f(G)=f(G \oplus \mathrm{i})-f(G), \quad S_{2} f(G)=f(G \oplus \mathrm{l})-f(G),
$$

where $\mathbf{i}$ is an isthmus (a one point rank one geometry) and 1 is a loop (a point rank zero pregeometry). To describe the range $S$ of $\mathrm{s}$, let $G \in \mathrm{C}$ and $p \in G$ be a point which is not a one point direct summand of $G$. Then all the functions $\mathrm{s}_{i, j}$, hence all the functions $f \in S$, satisfy

$$
f(G)=f(G-p)+f(G / p),
$$

where $G-p$ is the deletion of $p$ from $G$, and $G / p$ is the contraction of $G$ by $p$. The reader is referred to [2] for the details. The functions satisfying the identities (8) are called Tutte invariants and include the Möbius function $\mu(G)$, the chromatic polynomial $\lambda(G)$, and many others (see the table in [2, p. 253]). 
(5) Pólya's Hauptsatz. The expansions associated with the cycle index p: $(\mathbf{G}, s) \rightarrow(\mathrm{Pn}, s)$ are naturally involved with the circle of ideas surrounding Pólya's enumeration theorem (described by Read in [12]). The differential operators for the cycle index satisfy the product rule $P_{\lambda_{1}}=P_{\lambda_{1}} P_{\lambda_{2}} \cdots P_{\lambda_{r}}$ when $\lambda=\left(\lambda_{1} \lambda_{2} \cdots \lambda_{r}\right)$. Thus it suffices for applications to determine the operators corresponding to the one part partitions. As in the earlier examples we shall express $P_{n}$ in terms of shifts. Let $S_{n}$ denote the symmetric group on a set of $n$ elements. Using the cycle index of $S_{n}$,

$$
\mathbf{p}\left(S_{n}, \lambda\right)= \begin{cases}1 / \lambda ! \pi(\lambda) & \text { if }\|\lambda\|=n, \\ 0 & \text { if }\|\lambda\| \neq n,\end{cases}
$$

and letting $E_{n}$ denote the shift operator on $I(G)$ by $S_{n}$, we obtain, using (2.8),

$$
\begin{aligned}
E_{n} \mathrm{p}_{\lambda}(G) & =\mathrm{p}_{\lambda}\left(G \oplus S_{n}\right)=\sum_{\lambda \rightarrow \mu \oplus \nu} \mathrm{p}_{\mu}(G) \mathrm{p}_{\nu}\left(S_{n}\right) \\
& =\sum_{\|\nu\|=n} \frac{\mathrm{p}_{\lambda \ominus \nu}(G)}{\nu ! \pi(\nu)}=\sum_{\|\nu\|=n} \frac{P_{\nu} \mathrm{p}_{\lambda}(G)}{\nu ! \pi(\nu)} \\
& =\sum_{\|\nu\|=n} \frac{1}{r_{1} ! r_{2} ! \cdots}\left(\frac{P_{1}}{1}\right)^{r_{1}}\left(\frac{P_{2}}{2}\right)^{r_{2}} \cdots\left(\frac{P_{n}}{n}\right)^{r_{n}} \mathrm{p}_{\lambda}(G),
\end{aligned}
$$

where $\nu=\left(1^{r_{1}}, 2^{r_{2}}, \cdots, n^{r_{n}}\right)$. This we may invert by a standard generating function argument, which because of its importance in connection with symmetric functions we reproduce in full (see Riordan [13, p. 175]). Introducing the indeterminant $y$, we have

$$
\begin{aligned}
1+y E_{1}+y^{2} E_{2}+\cdots & =\sum_{r_{1}, r_{2}, \cdots=0}^{\infty} \frac{\left(y Q_{1}\right)^{r_{1}}}{r_{1} !} \frac{\left(y^{2} Q_{2}\right)^{r_{2}}}{r_{2} !} \cdots \frac{\left(y^{n} Q_{n}\right)^{r_{n}}}{r_{n} !} \\
& =\exp \left(y Q_{1}+y^{2} Q_{2}+\cdots+y^{n} Q_{n}+\cdots\right)
\end{aligned}
$$

where $Q_{n}=P_{n} / n$. Then

$$
\begin{aligned}
y Q_{1}+y^{2} Q_{2}+\cdots+y^{n} Q_{n}+\cdots & =\ln \left(1+y E_{1}+\cdots+y^{n} E_{n}+\cdots\right) \\
& =\sum_{\mu \in \mathrm{Pn}} \frac{1}{|\mu|}(-1)^{|\mu|-1} b(\mu) E_{\mu} y^{\|\mu\|} .
\end{aligned}
$$

Setting corresponding coefficients equal we obtain,

$$
\frac{P_{n}}{n}=\sum_{\|\mu\|=n} \frac{(-1)^{|\mu|-1} b(\mu) E_{\mu}}{|\mu|} .
$$


We should now, by analogy with the preceding examples, seek a family of identities characterizing the range $P$ of the morph $\mathbf{p}$. This we have not been able to do. It is partly with the hope of encouraging someone to find such a characterization that we have included this example. Without a usable characterization of $P$, our expansions have only a formal significance; that is they suggest results but do not by themselves prove them. Let us outline how by an application of the first expansion theorem we may formally obtain the conclusion of Pólya's Hauptsatz. Let $R=\left\{x_{1} x_{2} \cdots x_{m}\right\}$ be a fixed finite set. Each permutation group $G \in \mathbf{G}$ has an induced action, on the space of functions $\phi$ from $S_{G}$ to $R$, defined by $(g \phi)(a)=\phi\left(g^{-1}(a)\right)$ for $g \in G, a \in S_{G}$. For each function $\phi$ we have a monomial $\Pi \phi(a)$, the product extending over $a \in S_{G}$. Let $f(G)$ denote the sum of these monomials, one for each equivalence class of functions under the induced action of $G$. Now $f$ is not a real-valued function on $\mathbf{G}$, but one easily verifies that our theory has made no special use of the real numbers; in effect our functions can take values in any ring. So we may expand formally,

$$
\begin{aligned}
f(G)= & \sum_{\lambda \in \mathrm{Pn}}\left\langle P_{\lambda}, f\right\rangle \mathbf{p}_{\lambda}(G) \\
= & \sum_{\lambda=\left(1^{r_{1}, 2} r_{2}, \cdots\right)}{ }^{\left(x_{1}+x_{2}+\cdots+x_{m}\right)^{r_{1}}} \\
& \cdot\left(x_{1}^{2}+x_{2}^{2}+\cdots+x_{m}^{2}\right)^{r_{2}} \cdots \mathrm{p}_{\lambda}(G),
\end{aligned}
$$

which is the desired conclusion; however, we postpone until $\S 7$ the verification in detail that

$$
\left\langle P_{\lambda}, f\right\rangle=\left(x_{1}+\cdots+x_{m}\right)^{r_{1}}\left(x_{1}^{2}+\cdots+x_{m}^{2}\right)^{r_{2}} \cdots .
$$

4. Connection constants. Let $(S, m)$ and $(T, n)$ be dissects as in $\S 3$. Let $\mathbf{p}$ and $q$ be two morphs from $(S, m)$ to $(T, n)$ with the same range $P$. Then there are the following expansions:

$$
\mathbf{p}_{a}=\sum_{b \in \mathbf{T}} \mathbf{f}(b, a) \mathbf{q}_{b}, \quad \mathbf{q}_{a}=\sum_{b \in \mathbf{T}} \mathbf{g}(b, a) \mathbf{p}_{b},
$$

and

$$
P_{a}=\sum_{b \in \mathrm{T}} \mathrm{h}(a, b) Q_{b}, \quad Q_{a}=\sum_{b \in \mathrm{T}} \mathbf{k}(a, b) P_{a} .
$$

The coefficients of these expansions are called connection constants. We first observe that $h$ is a morph from $(T, n)$ to itself. This follows from substituting (2) into (M) and equating coefficients of $Q_{b}$. From $\mathrm{h}(a, b)=\left\langle P_{a}, \mathrm{q}_{b}\right\rangle=$ $\mathbf{g}(a, b)$, it follows that $\mathbf{h}=\mathbf{g}$; hence $\mathbf{g}$ is also a morph, in fact a morphism. 
Similarly, $\mathbf{f}=\mathbf{k}$ and, since these are clearly inverse to $\mathbf{h}=\mathbf{g}$, it follows that the connection constants are even automorphisms of $(T, n)$.

Definition 7. Let $\mathbf{p}$ be a morph from $(\mathrm{S}, m)$ to $(\mathrm{T}, n)$ with range $P$. An operator $U$ on $P$ is called umbral if $\mathbf{q}_{a}=U_{\mathbf{p}_{a}}$ is another morph with range $P$. The umbral operators are thus the operators on $P$ whose matrix with respect to the basis $\left\{\mathbf{p}_{a}\right\}$ is an automorphism of $(T, n)$. That this notion does not depend on the basis $\left\{\mathbf{p}_{a}\right\}$ is shown by the following

AUTOMORPHISM THEOREM. The following are equivalent for an operator $U$ on $P$.

(a) $U$ is an umbral operator.

(b) $U$ is invertible, the map $T \rightarrow U T U^{-1}$ is an automorphism of $P^{*}$, and

$$
\langle U T, f\rangle=\langle T, f\rangle, \quad T \in P^{*}, f \in P .
$$

(c) If $\mathrm{r}$ is any morph from $(\mathrm{S}, m)$ to $(\mathrm{T}, n)$ with range $\mathrm{P}$, and $\mathrm{s}_{a}=$ $U \mathbf{r}_{a}(a \in \mathrm{T})$, then $\mathrm{s}_{a}$ is also a morph from $(\mathrm{S}, m)$ to $(\mathrm{T}, n)$ with range $P$.

Proof. (a) $\Rightarrow$ (b). Let $U$ be an umbral operator and let $\mathrm{q}_{a}=U \mathrm{p}_{a} . U$ is invertible, since by definition $\left\{q_{a} \mid a \in T\right\}$ is a basis for $P$. Consider the operators $U P_{a} U^{-1}$. We have

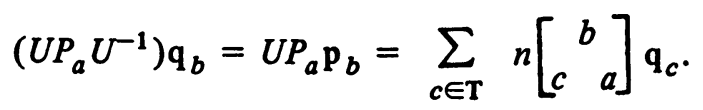

Thus $U P_{a} U^{-1}=Q_{a}$. By linearity the mapping $T \rightarrow U T U^{-1}$ then takes $P^{*}$ to $P^{*}$; it is clearly an automorphism. Finally we have

$$
\left\langle U P_{a}, \mathbf{p}_{b}\right\rangle=\left\langle U P_{a} U^{-1}, U \mathbf{p}_{b}\right\rangle=\left\langle Q_{a}, \mathbf{q}_{b}\right\rangle=\delta_{a, b}=\left\langle P_{a}, \mathbf{p}_{b}\right\rangle
$$

which extends by linearity to (3).

(b) $\Rightarrow$ (c). Let $\mathbf{r}$ be as given. Then the $s_{a}=U \mathbf{r}_{a}$ form a basis for $P$ since $U$ is invertible. The operators $U R_{a} U^{-1}$ are a dual basis since $\left\langle U R_{a} U^{-1}\right.$, $\left.\mathrm{s}_{b}\right\rangle=\left\langle R_{a}, \mathrm{r}_{b}\right\rangle=\delta_{a, b}$. Furthermore the operators $U R_{a} U^{-1}$ satisfy the same product rule as the operators $R_{a}$ themselves, since the map $T \rightarrow U T U^{-1}$ is an automorphism. It follows from the dual basis theorem that $s$ is a morph from $(\mathrm{S}, m)$ to $(\mathrm{T}, n)$ with range $\mathrm{P}$.

To summarize, the reader will have no trouble supplying the missing implications in the following

Proposition. The following groups are isomorphic:

(a) the group of umbral operators on $P$,

(b) the group of automorphisms of $P^{*}$,

(c) the group of automorphisms of $(T, n)$. 
Two morphs $\mathbf{q}$ and $\mathbf{r}$ from $(\mathbf{S}, m)$ to $(\mathbf{T}, n)$ are called inverse with respect to $\mathbf{p}$ when all these morphs have the same range and the umbral operator defined by $U \mathbf{p}_{a}=\mathbf{q}_{a}$ satisfies $U^{-1} \mathbf{p}_{a}=\mathbf{r}_{a}$.

SUMMATION THEOREM. Let $\mathrm{q}$ and $\mathrm{r}$ be inverse with respect to $\mathrm{p}$. Then

$$
\mathbf{r}_{a}=\sum_{b \in \mathbf{T}}\left\langle Q_{b}, \mathbf{p}_{a}\right\rangle \mathbf{p}_{b} .
$$

PRoof. By the first expansion theorem $\mathbf{p}_{a}=\Sigma_{b \in T}\left\langle Q_{b}, \mathbf{p}_{a}\right\rangle \mathbf{q}_{b}$. Now apply $U^{-1}$.

EXAMPLES. (1) The reader will find many examples of connection constants in [10] and [14]. Here is just a token. Two morphs from $(Z, s)$ to $(Z, b)$ will be connected by an automorphism of $(Z, b)$. Thus the Stirling numbers of the first kind $\mathrm{s}(k, n)$ defined by $(x)_{n}=\Sigma_{k} \mathrm{~s}(k, n) x^{k}$ satisfy the identity

$$
\left(\begin{array}{c}
k+j \\
k
\end{array}\right) \mathrm{s}(k+j, n)=\sum_{i=0}^{n}\left(\begin{array}{c}
n \\
i
\end{array}\right) \mathrm{s}(k, i) \mathrm{s}(j, n-i)
$$

for all $k, j$, and $n$. The group of all automorphisms of $(\mathrm{Z}, b)$ turns out not surprisingly to be isomorphic to the group of all exponential generating functions invertible under composition. This isomorphism is given by the mapping $\mathbf{s} \rightarrow$ $\Sigma_{n} s(1, n) x^{n} / n !$.

(2) The Tutte polynomial. Consider the subgeometry generating function s. We wish to find a second morph $t$ from $(C, s)$ to $\left(Z^{2}, s\right)$ with the same range $S$ but simpler differential operators. It would be a great advantage for example if the differential operator $T_{j, k}$ was simply the shift $T_{j, k} f(G)=$ $f\left(G \oplus \mathrm{i}^{j} \oplus \mathrm{l}^{k}\right)$, where $\mathrm{i}^{j} \oplus \mathrm{l}^{k}$ represents the direct sum of $j$ isthmi and $k$ loops. If the morph $t$ exists its differential operators $T_{1}=T_{10}, T_{2}=T_{01}$ are then related to the operators $S_{1}, S_{2}$ by $S_{1}=T_{1}-I, S_{2}=T_{2}-I$. This would imply that

$$
S_{m, n}=S_{1}^{m} S_{2}^{n}=\sum_{j, k}(-1)^{m+n+j+k}\left(\begin{array}{c}
m \\
j
\end{array}\right)\left(\begin{array}{l}
n \\
k
\end{array}\right) T_{i, k}
$$

so that

$$
\mathbf{t}_{m, n}(G)=\sum_{j, k}(-1)^{m+n+j+k}\left(\begin{array}{c}
m \\
j
\end{array}\right)\left(\begin{array}{l}
n \\
k
\end{array}\right) \mathbf{s}_{j, k}(G) .
$$

This last we may take as the definition of $t$. It is clear that $\mathbf{t}$ has the desired property since its connection constants with $\mathbf{s}$ are given by the automorphism $(-1)^{m+n+j+k}\left(\begin{array}{c}m \\ j\end{array}\right)\left(\begin{array}{l}n \\ k\end{array}\right)$ of $\left(\mathrm{Z}^{2}, s\right)$. The numbers $\mathrm{t}_{m, n}(G)$ are the coefficients of the Tutte polynomial (see [2]), a polynomial in two variables defined by 


$$
T(G)=\sum_{m, n} t_{m, n}(G) z_{1}^{m} z_{2}^{n} .
$$

An important notion in this connection is that of a Tutte bi-invariant. These are functions $f$ on $\mathbf{C}$ which, in addition to being Tutte invariants, that is satisfying (3.8), also satisfy $f\left(G_{1} \oplus G_{2}\right)=f\left(G_{1}\right) f\left(G_{2}\right), G_{1}, G_{2} \in \mathrm{C}$. We can easily prove the theorem that all Tutte bi-invariants are evaluations of the Tutte polynomial for we can expand $f$ according to $\S 3$, obtaining $\left\langle T_{m, n}, f\right\rangle=f\left(\mathrm{i}^{m} \oplus 1^{n}\right)=$ $f(i)^{m} f(1)^{n}$ so that

$$
f(G)=\sum_{m, n}\left\langle T_{m, n}, f\right\rangle t_{m, n}(G)=\left.T(G)\right|_{z_{1}=f(i) ; z_{2}=f(1)}
$$

Analogous considerations hold with regard to rook polynomials and order polynomials. In the latter case the morph $e$ already has shifts for its differential operators.

Returning to pregeometry we wish to point out that the operation $G \rightarrow G^{*}$ of forming the dual pregeometry induces an umbral operator $U$ on $S, U f(G)=$ $f\left(G^{*}\right), f \in S$. That $U$ in fact maps $S$ into $S$ follows from the relations $(G / p)^{*}=G^{*}-p$ and $(G-p)^{*}=G^{*} / p$. From $U T_{1} U^{-1}=T_{2}$ and $U T_{2} U^{-1}$ $=T_{1}$ we deduce that $U \mathrm{t}_{m, n}=\mathrm{t}_{n, m}$, thus $U$ is an umbral operator.

(3) Almost umbral operators. A number of operators $U$ appear in our examples, in connection with constructions of various types, which possess the property in common with umbral operators that when $\left\{\mathbf{p}_{a}\right\}$ defines a morph so does $\left\{U_{\mathbf{p}_{a}}\right\}$, but which fail to be umbral by not being invertible. For example let $H$ be a fixed permutation group and consider the mapping $G \rightarrow G \times H$ for $G \in \mathbf{G}$ and its induced mapping on $I^{*}(\mathbf{G})$. Here $G \times H$ is the usual product of permutation groups, the direct product of the groups $G$ and $H$ acting componentwise on the cartesian product $S_{G} \times S_{H}$. Let $\mathrm{p}_{\lambda}$ be the cycle index morph from $(\mathrm{G}, s)$ to $(\mathrm{Pn}, s)$, and set $\mathrm{q}_{\lambda}(G)=U_{\mathrm{p}_{\lambda}}(G)=\mathrm{p}_{\lambda}(G \cdot \times H)$. From the distributive law $\left(G_{1} \oplus G_{2}\right) \times H=\left(G_{1} \times H\right) \oplus\left(G_{2} \times H\right)$ follows

$$
\mathbf{q}_{\lambda}\left(G_{1} \oplus G_{2}\right)=\sum_{\lambda=\lambda_{1} \oplus \lambda_{2}} \mathbf{q}_{\lambda_{1}}\left(G_{1}\right) \mathbf{q}_{\lambda_{2}}\left(G_{2}\right) \text {. }
$$

Thus $q$ is also a morph from (G, $s$ ) to $(\mathrm{Pn}, s)$. The range of $q$ is not all of $P$, and $q$ is not a monomorph since for example $q_{\lambda} \equiv 0$ whenever $\|\lambda\|<\left|S_{H}\right|$. Thus $U$ is not an umbral operator. We still have connection constants

$$
\mathbf{q}_{\mu}=\sum_{\lambda} \mathbf{f}(\lambda, \mu) \mathbf{p}_{\lambda}, \quad \mathbf{f}(\lambda, \mu)=\left\langle P_{\lambda}, \mathbf{q}_{\mu}\right\rangle
$$

and $\mathrm{f}$ is a morphism of $(\mathrm{Pn}, s)$ to itself as follows from substituting (6) into (5) and equating coefficients. Other constructions which, like the product of 
permutation groups, satisfy a distributive law, and so induce almost umbral operators, include the product of posets in $\mathbf{P}$ and the krantz product in $\mathbf{G}$. Because these do not lead to invertible operators, we cannot exploit the duality of operators and functions in these examples.

\section{Double dissects.}

Definition 8. A double dissect is a set $\mathbf{S}$ and two sets of incidence coefficients $n$ and $n^{\prime}$ both satisfying the conditions of Definition 1. We will usually assume that $n$ has an identity, but not necessarily $n^{\prime}$. If (S, $\left.n, n^{\prime}\right)$ and (T, $\left.m, m^{\prime}\right)$ are two double dissects, a morph [morphism, ${ }^{*}$ morphism] p: $\left(\mathbf{S}, n, n^{\prime}\right)$ $\rightarrow\left(\mathbf{T}, m, m^{\prime}\right)$ is a real-valued function $\mathbf{p}$ defined on $\mathbf{S} \times \mathbf{T}$ such that $\mathbf{p}$ is a morph [morphism, *morphism] from $(\mathbf{S}, m)$ to $(\mathbf{T}, n)$ and a morph [ ${ }^{*}$ morphism, morphism] from $\left(T, n^{\prime}\right)$ to $\left(\mathrm{S}, m^{\prime}\right)$. Thus in addition to (B), p satisfies the identity

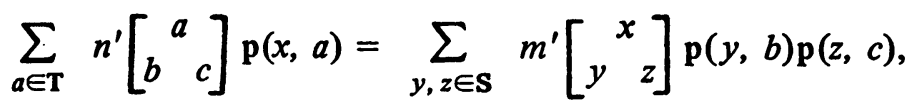

for all $b, c \in \mathbf{T}, x \in \mathbf{S}$. ( (') $^{\prime}$ may be rewritten

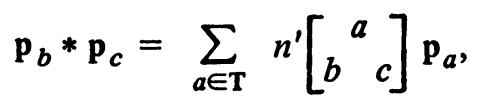

where the convolution on the left is with respect to the incidence coefficients $m^{\prime}$. Thus $P$ is a subalgebra of $I^{*}\left(S, m^{\prime}\right)$.

Let $\mathbf{p :}\left(\mathbf{S}, m, m^{\prime}\right) \rightarrow\left(\mathrm{T}, n, n^{\prime}\right)$ be a morph. By definition the elements of $P^{*}$ are operators on $P$. We can now define conversely an action of $P$ on $P^{*}$ by taking the adjoint of (left) multiplication. In other words, for $f \in P$, $Q \in P^{*}$, we define $f(Q)$ to be the operator of $P^{*}$ such that

$$
\langle f(Q), g\rangle=\langle Q, g * f\rangle, \quad g \in P .
$$

Of course $f$ is a linear operator on $P^{*}$. Furthermore the association of $f \in P$ with the operator $f$ on $P^{*}$ is an algebra homomorphism, that is $(g * f)(Q)=$ $g(f(Q))$. By an application of the second expansion theorem we find that

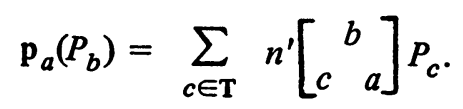

Thus $\mathbf{p}_{a}$ acts on the operator $P_{b}$ in a manner analogous to the original definition of differential operators but with the coefficients $n^{\prime}$ in place of $n$. Further developments now depend on postulating some connection between these two incidence coefficients.

Definition 9. Let (T, $\left.n, n^{\prime}\right)$ be a double dissect. (a) $\mathrm{T}$ is a Hopf dissect if, for all $a, b, c, d \in \mathrm{T}$, 


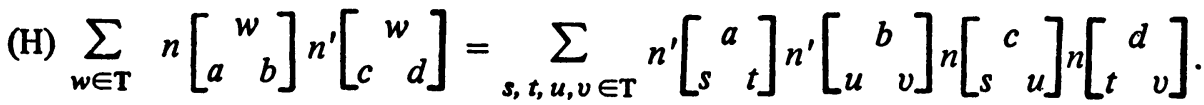

(b) $\mathrm{T}$ has a double identity $e$ if $e$ is an identity for both $n$ and $n^{\prime}$, and (c) let $\lambda$ be a real-valued function on $T ; \lambda$ is an inverse if $T$ has a double identity and

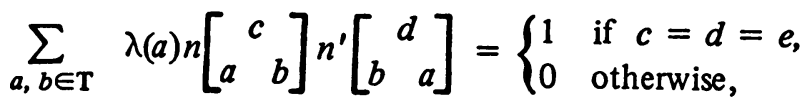

and $\lambda$ is a post if

$$
\lambda(a) \lambda(b)=\lambda(c) \text { when } n\left[\begin{array}{ll}
c \\
a & b
\end{array}\right] \neq 0 .
$$

To avoid triviality we also assume $\lambda(a) \neq 0$ for all $a \in \mathbf{T}$.

Let $\mathrm{p}:\left(\mathrm{S}, m, m^{\prime}\right) \rightarrow\left(\mathrm{T}, n, n^{\prime}\right)$ be a morph. If (T, $\left.n, n^{\prime}\right)$ is a Hopf dissect, then we have the Leibnitz formulae,

$$
\begin{array}{ll}
P_{c}(g * f)=\sum_{a, b \in \mathbf{T}} n^{\prime}\left[\begin{array}{cc}
c \\
a & b
\end{array}\right] P_{a}(g) P_{b}(f), \quad g, f \in P, \\
\mathbf{p}_{c}(Q R)=\sum_{a, b \in \mathbf{T}} n\left[\begin{array}{cc}
c \\
a & b
\end{array}\right] \mathbf{p}_{a}(Q) \mathbf{p}_{b}(R), & Q, R \in P^{*} .
\end{array}
$$

To prove (L) it suffices to consider the case where $g$ and $f$ come from the basis $\left\{p_{a} \mid a \in T\right\}$. The proof is completed by the computation $\left(M^{\prime}\right)(D)^{*}(H)^{*}\left(M^{\prime}\right)(D)(D)$. $\left(L^{\prime}\right)$ is proved using the dual computation $(M)\left(D^{\prime}\right)^{*}(H)^{*}(M)\left(D^{\prime}\right)\left(D^{\prime}\right)$. Let $\lambda$ be a real-valued function on $T$. We shall use $\lambda$ to construct negative shift operators. For this purpose it is useful to imagine a copy of the set $\mathbf{S}$. The elements of this copy of $\mathbf{S}$ will be called the negatives of the elements of $\mathbf{S}$; for each $x \in \mathrm{S}$, its negative will be written $-x$. We begin by defining values for the functions $\mathbf{p}_{a}$ at the negatives by setting

$$
\mathbf{p}_{a}(-x)=\lambda(a) \mathbf{p}_{a}(x)
$$

Then we define the negative shift operator $E_{-x}$ by

$$
E_{-x} \mathbf{p}_{a}=\sum_{b, c \in T} n\left[\begin{array}{c}
a \\
b
\end{array}\right] \mathbf{p}_{b}(-x) \mathbf{p}_{c}
$$

and extending by linearity to all $f \in P$. We can then define values for all $f \in P$ on the negatives by

$$
\left\langle E_{-x}, f\right\rangle=f(-x)
$$

which amounts to extending (2) by linearity. Then we also have 


$$
E_{-x} f=\sum_{c \in \mathbf{T}} P_{c} f(-x) \mathbf{p}_{c} .
$$

Supposing that $\lambda$ is a post, the negative shifts satisfy the product rule

$$
E_{-x} E_{-y}=\sum_{z \in S} m\left[\begin{array}{cc}
z \\
y & x
\end{array}\right] E_{-z}
$$

This is proven by applying the left side to the typical element $\mathbf{p}_{a}$ of $P$ and using $(E)(E)^{*}(J)(A)^{*}(B)^{*}(E)$. It is worth noting that the negative shifts behave exactly like the ordinary shifts. A related consequence of $(J)$ is that if we let $L$ be the operator of negation, $L f(x)=f(-x)$, then $L$ is an umbral operator. Passing to consequences of (I), suppose that $\lambda$ is an inverse (but not necessarily a post). The most important property of the negative shifts is the following identity. For each $x \in \mathrm{S}$,

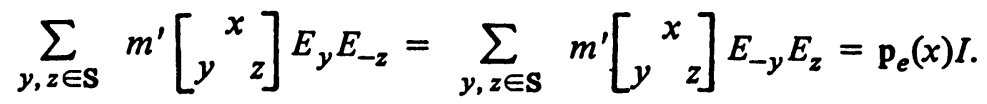

The proof arises by applying the left side to $p_{a}$ and using $(E)(E)^{*}(A)^{*}\left(B^{\prime}\right)^{*}(I)$. In applications the incidence coefficients $m^{\prime}$ are usually the trivial coefficients of pointwise multiplication. Then the left side of $(\mathrm{N})$ reads $E_{x} E_{-x}$. Now $\mathrm{p}_{e}(x)$ can be only a zero or a one (apply (B) with $a=e$ ). In the former case $E_{-x}$ is a genuine inverse to $E_{x}$ and $(\mathrm{N})$ yields inverse relations, in the latter case we have an orthogonality relation. Finally we have a closed form for the adjoint action defined in (1). For $f \in P, Q \in P^{*}$

$$
f(Q)=\sum_{a \in \mathrm{T}}\left(L P_{a} f\right) Q\left(\mathrm{p}_{a}\right),
$$

where by $\left(L P_{a} f\right) Q\left(\mathrm{p}_{a}\right)$ we mean the operator composed of left multiplication by $\mathrm{p}_{a}$, followed by $Q$, followed by left multiplication by $L P_{a} f$. Note that the series in $\left(F^{\prime}\right)$ is finite since $f$ is annihilated by all but a finite number of $P_{a}$. For the proof it suffices to examine the right-hand side of $\left(\mathrm{F}^{\prime}\right)$ for the case $f=\mathrm{p}_{b}, T=P_{c}$ and the operator signified is applied to $\mathrm{p}_{d}$. The result then follows from the computation $\left(M^{\prime}\right)(D)(D)\left(M^{\prime}\right)^{*}(H)^{*}(A)\left(A^{\prime}\right)^{*}(I) *(D)\left(D^{\prime}\right)$.

EXAMPLES. (1) Let us interpret these results for the polynomial sequence $\mathrm{d}_{n}=x^{n}$ as a morph from $(\mathrm{Z}, s)$ to $(\mathrm{Z}, b)$. We take the trivial incidence coefficients as the second set for $(Z, s)$. Then $d$ is a morph from $(Z, s, p)$ to $(\mathrm{Z}, b, s) ;\left(\mathrm{M}^{\prime}\right)$ in this case reduces to $x^{n} x^{m}=x^{n+m}$. The adjoint action is determined by the action of $x$ on the shift invariant operators. We will always write $T^{\prime}$ for $x(T), T \in D^{*}$. The identity (D) gives $D_{n}^{\prime}=D_{n-1}$ or $\left(D^{n} / n !\right)^{\prime}$ $=D^{n-1} /(n-1)$ !; thus $x$ acts like ordinary differentiation with respect to $D$. This is the Pincherle derivative as used by Mullin and Rota. 
$(\mathrm{Z}, b, s)$ is a Hopf dissect. The formula $(\mathrm{H})$ is the Vandermonde convolution; while (L) is the usual Leibnitz formula. $(\mathrm{Z}, b, s)$ also has a double identity (zero) and an inverse $\lambda(n)=(-1)^{n}$; the identity (I) reducing in the most important case $(c=d)$ to the identity

$$
\sum_{k}(-1)^{k}\left(\begin{array}{l}
c \\
k
\end{array}\right)=\left\{\begin{array}{lll}
1 & \text { if } c=0 \\
0 & \text { if } c>0
\end{array}\right.
$$

The negative values defined by (2) are the usual values of $x^{n}$ at negative integers. Finally $\left(\mathrm{F}^{\prime}\right)$ yields the formula $T^{\prime}=T x-x T$, which in [10] serves as the definition of the Pincherle derivative. At this point we can easily derive the results of [10] on closed forms of polynomials and the Rodrigues formula.

RODRIGUES FORMULA. Let $\mathbf{q}_{n}$ be a polynomial sequence of binomial type with delta operator $Q$. Then $Q^{\prime}$ is invertible and $\mathbf{q}_{n}=x\left(Q^{\prime}\right)^{-1} \mathbf{q}_{n-1}$ for $n \geqslant 1$.

Proof. The expansion of $Q$ in terms of $D$,

$$
Q=\sum_{n \geq 0}\left\langle Q, x^{n}\right\rangle D^{n} / n !
$$

has no constant term since $Q 1=0$, but a nonzero linear term since $Q x$ is a nonzero constant. Thus the expansion for $Q^{\prime}$,

$$
Q^{\prime}=\sum_{n \geqslant 0}\left\langle x(Q), x^{n}\right\rangle D^{n} / n !=\sum_{n \geq 0}\left\langle Q, x^{n+1}\right\rangle D^{n} / n !
$$

has a nonzero constant term. Thus, by the isomorphism between $D^{*}$ and the algebra of exponential generating functions, $Q^{\prime}$ is invertible. We shall prove the Rodrigues formula by showing that $\left\langle Q_{m}, x\left(Q^{\prime}\right)^{-1} \mathbf{q}_{n-1}\right\rangle=\left\langle Q_{m}, \mathbf{q}_{n}\right\rangle$ for all $m \geqslant 0$, where $Q_{m}=Q^{m} / m$ ! is the dual basis of $\mathbf{q}_{n}$. By linearity it will follow that $\left\langle T, x\left(Q^{\prime}\right)^{-1} \mathbf{q}_{n-1}\right\rangle=\left\langle T, \mathbf{q}_{n}\right\rangle$ for all $T \in D^{*}$, hence that $x\left(Q^{\prime}\right)^{-1} \mathbf{q}_{n-1}=$ $\mathbf{q}_{n}$. The case where $m=0$ is trivial. For $m \geqslant 1$,

$$
\begin{aligned}
\left\langle Q_{m}, x\left(Q^{\prime}\right)^{-1} \mathbf{q}_{n-1}\right\rangle & =\left\langle Q_{m}^{\prime},\left(Q^{\prime}\right)^{-1} \mathbf{q}_{n-1}\right\rangle \\
& =\left\langle Q_{m-1} Q^{\prime},\left(Q^{\prime}\right)^{-1} \mathbf{q}_{n-1}\right\rangle=\left\langle Q_{m-1}, \mathbf{q}_{n-1}\right\rangle=\left\langle Q_{m}, \mathbf{q}_{n}\right\rangle .
\end{aligned}
$$

CLOSED FORMS. With the same hypothesis as the previous result, we may factor $D$ from the expansion (3) of $Q$, obtaining $Q=D P$, where $P$ is an invertible shift invariant operator. Then for $n \geqslant 1$ we have
(a) $\mathbf{q}_{n}=Q^{\prime} P^{-n-1} x^{n}$,
(b) $\mathrm{q}_{n}=P^{-n} x^{n}-\left(P^{-n}\right)^{\prime} x^{n-1}$,
(c) $\mathrm{q}_{n}=x P^{-n} x^{n-1}$. 
Proof. As in [10] we begin by showing that all three formulae give the same polynomial sequence.

$$
\begin{aligned}
Q^{\prime} P^{-n-1} x^{n} & =(D P)^{\prime} P^{-n-1} x^{n}=\left(P+D P^{\prime}\right) P^{-n-1} x^{n} \\
& =P^{-n} x^{n}-n^{-1}\left(P^{-n}\right)^{\prime} D x^{n}=P^{-n} x^{n}-\left(P^{n}\right)^{\prime} x^{n-1} \\
& =P^{-n} x^{n}-\left(P^{-n} x-x P^{-n}\right) x^{n-1}=x P^{-n} x^{n-1} .
\end{aligned}
$$

We can now establish simultaneously the equality of all three with $\mathbf{q}_{n}$ by induction. For $n=1, x P^{-1} 1=x /\langle Q, x\rangle=q_{1}$. Assuming now that $\mathrm{q}_{n}=$ $Q^{\prime} P^{-n-1} x^{n}$, we shall prove that $\mathrm{q}_{n+1}=x P^{-n-1} x^{n}$ by showing that $Q_{m}$, $\left.x P^{-n-1} x^{n}\right\rangle=\left\langle Q_{m}, \mathbf{q}_{n+1}\right\rangle$ for all $m \geqslant 0$. As before this is trivial for $m=0$. For $m \geqslant 1$,

$$
\begin{aligned}
\left\langle Q_{m}, x P^{-n-1} x^{n}\right\rangle & =\left\langle\left(Q_{m}\right)^{\prime}, P^{-n-1} x^{n}\right\rangle=\left\langle Q_{m-1}, Q^{\prime} P^{-n-1} x^{n}\right\rangle \\
& =\left\langle Q_{m-1}, \mathbf{q}_{n}\right\rangle=\left\langle Q_{m}, \mathbf{q}_{n+1}\right\rangle .
\end{aligned}
$$

(2) Consider the morph $\mathbf{b}:(\mathbf{Z}, s) \rightarrow(\mathbf{Z}, s), \mathbf{b}(x, n)=\left(\begin{array}{l}x \\ n\end{array}\right)$. As in the preceding example we choose the incidence coefficients of pointwise multiplication and expand the product $\left(\begin{array}{l}x \\ m\end{array}\right)\left(\begin{array}{l}x \\ n\end{array}\right)$ in terms of $\left(\begin{array}{l}x \\ k\end{array}\right)$ obtaining,

$$
\left(\begin{array}{l}
x \\
m
\end{array}\right)\left(\begin{array}{l}
x \\
n
\end{array}\right)=\sum_{k} b\left[\begin{array}{cc}
k-m & k-n \\
k & m+n-k
\end{array}\right]\left(\begin{array}{l}
x \\
k
\end{array}\right) .
$$

Therefore b is a morph from $(\mathrm{Z}, s, p)$ to $(\mathrm{Z}, s, e)$ where $e\left[{ }_{m}^{k}{ }_{n}\right]$ is the multinomial coefficient $b\left[\begin{array}{c}k-m \\ k-n\end{array} \stackrel{k}{k}+n-k\right]$. The identity (4) is most easily proven by noting its combinatorial interpretation counting the number of different ways of choosing a pair of sets of cardinalities $m$ and $n$ from a set of cardinality $x$ by first choosing the union of the two sets (cardinality $k$ ). We have already pointed out that the incidence coefficient $e\left[{ }_{m}{ }_{n}{ }_{n}\right]$ counts the number of ways of choosing sets of cardinalities $m$ and $n$ from a set of cardinality $k$ so as to exhaust the set of cardinality $k$. The double dissect $(\mathrm{Z}, s, e)$ is a Hopf dissect. This yields the following Leibnitz type identity for the forward difference operators,

$$
\Delta^{k}(f g)=\sum_{m, n} e\left[\begin{array}{cc}
k \\
m & n
\end{array}\right] \Delta^{m} f \Delta^{n} g
$$

In particular we get the familiar rule $\Delta(f g)=\Delta(f) g+f \Delta(g)+\Delta(f) \Delta(g)$. Since the incidence coefficients $e$ have no identity, $(\mathrm{Z}, s, e)$ can have no inverse.

(3) The inverse of Example 1 may be thought of as derived from the Möbius inversion formula on the lattice of finite subsets of a countable set, or rather on the reduced incidence algebra of this lattice (see [18] for a discussion of reduced incidence algebras and Möbius inversion, in particular Example 4.6, p. 281). 
Other examples are supplied by other reduced incidence algebras. For example the reduced incidence algebra of the lattice of finite-dimensional subspaces of a vector space of countable dimension over $G F(q)$ yields the dissect $(Z, g)$, where $g$ are the Gaussian coefficients [18, Example 4.9]. The Möbius function $\lambda(n)=$ $(-1)^{n} q^{C_{n, 2}}$ is an inverse for $(\mathrm{Z}, g, s)$. An example of a morph is given by the Gaussian polynomials $\mathrm{p}_{n}(x, z)=(x-z)(x-q z) \cdots\left(x-q^{n-1} z\right)$ which give a morph from $\left(Z^{*}, s\right)$ to $(Z, g)$, where $Z^{*}$ is the set of pairs $(x, y)$ with $x \leqslant y$, and $s$ denotes the usual incidence coefficients on $Z^{*}$ with respect to the dissections $(x, z) \rightarrow(x, y) \oplus(y, z)$, where $x \leqslant y \leqslant z$. That is, $\mathrm{p}$ satisfies the identity,

$$
\mathbf{p}_{n}(x, z)=\sum_{k}\left(\begin{array}{l}
n \\
k
\end{array}\right) \mathbf{p}_{q}(x, y) \mathbf{p}_{n-k}(y, z)
$$

Treating this example with some care, as $\mathbf{p}$ is not exactly a double morph, one can recover part of the results of Andrews [1] (but see the discussion of this point in §9).

6. Symmetric functions. Let $X=\left\{x_{1}, x_{2}, x_{3}, \cdots\right\}$ be an infinite sequence of indeterminants. Let $\mathbf{N}$ be the dissect of all finite subsets of $X$ with the dissections $A \rightarrow B \oplus C$ when $A$ is the disjoint union of $B$ and $C$. The importance of $\mathbf{N}$ is that all the major classes of symmetric functions may be viewed as morphs from $(\mathrm{N}, s, p)$, where $s$ is the usual set of incidence coefficients and $p$ are the trivial incidence coefficients on $\mathbf{N}$, to $\mathbf{P n}$ supplied with various pairs of incidence coefficients (unfortunately a different pair for almost every type of symmetric function). To reach this point of view we must consider morphs which as functions on $\mathbf{N} \times \mathbf{P n}$ are symmetric polynomial valued rather than real valued. The range $P$ in $I^{*}(N)$ for all these morphs will consist of those functions $f \in I^{*}(\mathrm{~N})$ for which $f(B)$ is a symmetric polynomial in the indeterminants of $B$, and when $B \subseteq A, f(B)$ is obtained from $f(A)$ by setting to zero the indeterminants of $A-B$. This algebra $P$ may be regarded as a device for studying symmetric functions in infinitely many variables without ever actually using infinitely many variables.

As in other recent treatments of symmetric functions, we shall emphasize their combinatorial interpretation. Each class of symmetric functions counts tableaux of some type. A tableau is an arrangement of positive integers in the shape of the Ferrer's graph of a partition. For example

$\begin{array}{llll}1 & 1 & 1 & 4 \\ 1 & 1 & 1 & \\ 2 & 3 & & \\ 1 & 4 & & \end{array}$


is a tableau of shape $\left(432^{2}\right)$. Within each row the numbers read in increasing order; thus the row is entirely determined by its contents. Each tableau contributes a monomial when counted by a symmetric polynomial. The tableau (1) will contribute the monomial $x_{1}^{6} x_{2}^{2} x_{3} x_{4}^{2}$. The tableaux may be placed in one-toone correspondence with matrices such that the row sums of the matrix give the shape of the corresponding tableau, while the column sums give the numbers of 1's, 2's and so forth in the tableau. Corresponding to (1) we have the matrix

$$
\left[\begin{array}{llll}
2 & 1 & 0 & 1 \\
3 & 0 & 0 & 0 \\
0 & 1 & 1 & 0 \\
1 & 0 & 0 & 1
\end{array}\right]
$$

Thus symmetric functions also count classes of matrices. Both tableaux and matrices may be also thought of as solutions to distribution problems where the positive numbers are balls of different types to be placed in boxes, called rows, of limited capacity.

The monomial symmetric functions. The polynomial $\mathrm{k}(A, \lambda)$ counts the tableaux of shape $\lambda$ with constant rows, no two the same with rows of the same length arranged in increasing order by subscript. In addition these tableaux must contain only the positive numbers which are subscripts of indeterminants of $A$. Thus

$$
k\left(\{3,5,6\}, 2^{2} 1\right)=x_{3}^{2} x_{5}^{2} x_{6}+x_{3}^{2} x_{5} x_{6}^{2}+x_{3} x_{5}^{2} x_{6}^{2}
$$

corresponding to the tableaux

$\begin{array}{ccc}33 & 33 & 55 \\ 55 & 66 & 66 \\ 6 & 5 & 3\end{array}$

k satisfies the identity

$$
\mathrm{k}(A \oplus B, \lambda)=\sum s\left[\begin{array}{cc}
\lambda \\
\lambda_{1} & \lambda_{2}
\end{array}\right] \mathrm{k}\left(A, \lambda_{1}\right) \mathrm{k}\left(B, \lambda_{2}\right),
$$

which counts in two ways the tableaux described above with entries from $A \oplus B$, since each such tableau can be obtained in one and only one way from a tableau with entries from $A$ and a tableau with entries from $B$. Furthermore since rows of these tableaux are constant, the shapes of the tableaux into which a given tableau of shape $\lambda$ decomposes must be such that $\lambda=\lambda_{1} \oplus \lambda_{2}$, whence the appear- 
ance of the usual incidence coefficients $s$ associated with the separations of $\lambda$. Thus $\mathbf{k}$ is a morph from $(\mathrm{N}, s)$ to $(\mathrm{Pn}, s)$.

The incidence coefficients $m\left[{ }_{\mu}^{\lambda}{ }_{\nu}\right]$ arising in the expansion of the product

$$
\mathbf{k}(A, \mu) \mathbf{k}(A, \nu)=\sum_{\lambda} m\left[\begin{array}{cc}
\lambda \\
\mu & \nu
\end{array}\right] \mathbf{k}(A, \lambda)
$$

count the number of ways the parts of $\mu$ and $\nu$ may be arranged (with parts of size zero added as necessary) so as to sum to $\lambda$. Thus, for example,

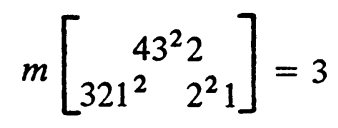

corresponding to the arrangements

$$
\begin{aligned}
& \begin{array}{lllllllllllll}
\mu & 3 & 1 & 1 & 2 & 2 & 3 & 1 & 1 & 2 & 1 & 3 & 1
\end{array} \\
& \text { v } 1220 \underline{2021} 2201 \\
& \lambda 443324433244332
\end{aligned}
$$

which give the different ways the term $x_{1}^{4} x_{2}^{3} x_{3}^{3} x_{4}^{2}$, for example, can arise as a product of terms which use the exponents $\left(321^{2}\right)$ in some order and the exponents $\left(2^{2} 1\right)$ in some order. Equivalently $m\left[{ }_{\mu \nu}{ }_{\nu}\right]$ counts the number of matrices with $|\lambda|$ rows and two columns with row sums $\lambda$ and columns consisting of the parts of $\mu$ and $\nu$. These numbers occur throughout the theory of symmetric functions. Summarizing (2) and (3), $\mathrm{k}$ is a morph from ( $\mathrm{N}, s, p)$ to (Pn, $s, m)$. $(\mathrm{Pn}, s, m)$ is a Hopf dissect. The identity $(\mathrm{H})$ in this case amounts to the assertion

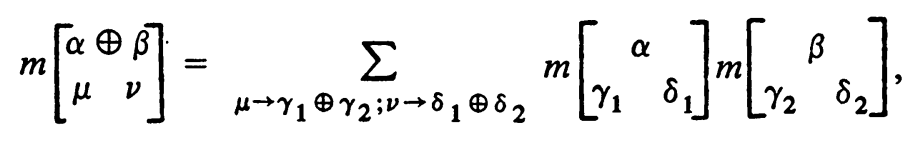

a sort of Vandermonde convolution for the incidence coefficients $m$ which has an obvious combinatorial interpretation in terms of the interpretation just given for $m$.

The idea of using differential operators in the investigation of symmetric functions is quite classical. These operators were most thoroughly developed by MacMahon who made them the cornerstone of his treatise Combinatory analysis [9]. The operators were previously the invention of Hammond (see the references in [9, p. 27]). The operators $K_{\lambda}$ satisfy the product rule $K_{\lambda}=K_{\lambda_{1}} K_{\lambda_{2}} \cdots$ when $\lambda=\left(\lambda_{1}, \lambda_{2}, \cdots\right)$, so that it suffices to describe the operators $K_{n}$ corresponding to one part partitions. These were termed obliterating operators by MacMahon and notated $D_{n}$ in $\left[9\right.$, p. 28] since the action of $K_{n}$ on a symmetric function $\mathrm{k}_{\lambda}$ is to remove one part $n$; in symbols $K_{n} \mathbf{k}_{\lambda}=\mathbf{k}_{\lambda \ominus n}$. 
The power sum symmetric functions. The polynomial $\mathrm{s}(A, \lambda)$ counts tableaux with constants rows with no restriction on the order of the rows and, in addition, identical rows permitted. $s$ satisfies the identities

$$
\begin{gathered}
\mathrm{s}(A \oplus B, \lambda)=\sum_{\mu, \nu} b\left[\begin{array}{cc}
\lambda \\
\mu & \nu
\end{array}\right] \mathrm{s}(A, \mu) \mathrm{s}(B, \nu), \\
\mathrm{s}(A, \mu) \mathrm{s}(A, \nu)=\mathrm{s}(A, \mu \oplus \nu),
\end{gathered}
$$

where the incidence coefficients $b\left[{ }_{\mu \nu}^{\lambda}\right]$ are zero unless $\lambda=\mu \oplus \nu$ when $b\left[{ }_{\mu \nu}^{\lambda}\right]=\lambda ! / \mu ! \nu$ !. Equation (4) is justified by considerations analogous to those just detailed for equation (2); the incidence coefficients $b$ arise by the interweaving of the rows of equal length from tableaux of shapes $\mu$ and $\nu$. Equation (5) merely reflects that the rows of these tableaux, unlike those counted by $k_{\lambda}$, may be chosen independently. On account of (5), $s$ is called a multiplicative set of symmetric functions. Thus $\mathbf{s}$ is a morph from $\mathbf{N}$ to $(\mathrm{Pn}, b, s) .(\mathrm{Pn}, b, s)$ is also a Hopf dissect.

The operators $S_{\lambda}$ satisfy the product rule

$$
b\left[\begin{array}{ccc}
\lambda_{1} & \lambda & \\
\lambda_{2} & \cdots & \lambda_{r}
\end{array}\right] S_{\lambda}=S_{\lambda_{1}} S_{\lambda_{2}} \cdots S_{\lambda_{r}},
$$

where $\lambda=\left(\lambda_{1}, \lambda_{2}, \cdots, \lambda_{r}\right)$. Thus as before it suffices to describe $S_{n}$. In addition we have the Leibnitz rule,

$$
S_{\lambda}(p q)=\sum_{\lambda=\mu \oplus \nu} S_{\mu}(p) S_{\nu}(q)
$$

In particular $S_{n}$ is a derivation. Therefore the $S_{\lambda}$ may be described by the equation $S_{n}\left(s_{m}\right)=\delta_{n, m}$.

The elementary symmetric functions. The polynomial $\mathrm{a}(A, \lambda)$ counts all tableaux with strictly increasing rows. Alternatively these tableaux correspond to matrices whose entries are zeros and ones. Thus

$$
\mathrm{a}(21,\{2,3,4\})=x_{2}^{2} x_{3}+x_{2} x_{3}^{2}+x_{2} x_{4}^{2}+x_{3}^{2} x_{4}^{2}+3 x_{2} x_{3} x_{4}
$$

corresponding to the tableaux

$\begin{array}{lllllllll}23 & 23 & 23 & 24 & 24 & 24 & 34 & 34 & 34 \\ 2 & 3 & 4 & 2 & 3 & 4 & 2 & 3 & 4\end{array}$

a satisfies the identities,

$$
\begin{gathered}
\mathbf{a}(A \oplus B, \lambda)=\sum_{\mu, \nu} m\left[\begin{array}{cc}
\lambda \\
\mu & \nu
\end{array}\right] \mathrm{a}(A, \mu) \mathbf{a}(B, \nu), \\
\mathbf{a}(A, \mu) \mathbf{a}(A, \nu)=\mathbf{a}(A, \mu \oplus \nu) .
\end{gathered}
$$


Equation (8) counts in two ways the tableaux of shape $\lambda$ with entries from $A \oplus B$. Each such tableaux may be decomposed into two tableaux, one with entries from $A$ and the other with entries from $B$. An example should make this clear. With $A=\{1,5,6\}, B=\{2,3,7,9\}$ and $\lambda=(54321)$, the tableau

$$
\begin{array}{lllll}
1 & 3 & 5 & 6 & 7 \\
2 & 3 & 5 & 9 & \\
2 & 6 & 9 & & \\
1 & 6 & & & \\
7 & & & &
\end{array}
$$

is initially divided into the two arrays of numbers

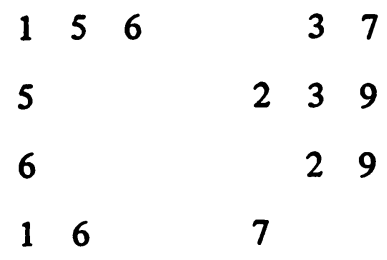

each of which is rearranged, keeping rows of equal length in the same order, to form a tableau. We obtain

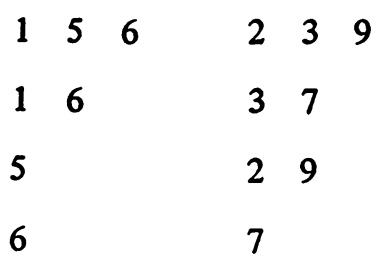

of shapes $\left(321^{2}\right)$ and $\left(32^{2} 1\right)$, respectively. The incidence coefficient $m$ arises because to a pair of tableaux of shapes $\mu$ and $\nu$, with entries from sets $A$ and $B$, respectively, there corresponds by this decomposition $m\left[{ }_{\mu}{ }_{\nu}\right]$ tableaux of shape $\lambda$. These correspond to the different ways the parts of $\mu$ and $\nu$-or rather the corresponding rows of the tableaux of shapes $\mu$ and $\nu$-can be added together to get $\lambda$. Continuing the above example, we have

$$
m\left[\begin{array}{cc}
54321 \\
321^{2} & 32^{2} 1
\end{array}\right]=3
$$

corresponding to the matrices 


$$
\left[\begin{array}{ll}
3 & 2 \\
1 & 3 \\
1 & 2 \\
2 & 0 \\
0 & 1
\end{array}\right]\left[\begin{array}{ll}
3 & 2 \\
1 & 3 \\
2 & 1 \\
0 & 2 \\
1 & 0
\end{array}\right]\left[\begin{array}{ll}
2 & 3 \\
3 & 1 \\
1 & 2 \\
0 & 2 \\
1 & 0
\end{array}\right] .
$$

Therefore there are exactly three tableaux of shape (54321) which, when decomposed as above, will yield the same pair of tableaux (11). One of these three is naturally the tableau (10) with which the example began. It corresponds to putting together the rows of the tableaux (11) according to the first of the above matrices. The other two tableaux are

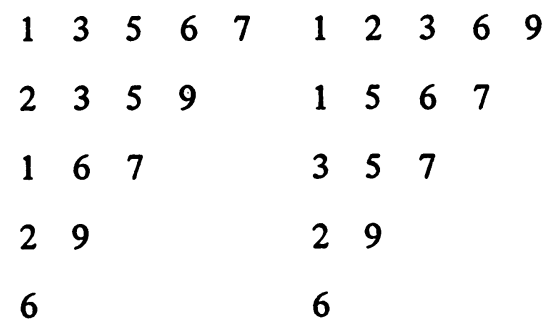

Thus a is a morph from $\mathbf{N}$ to $(\mathrm{Pn}, m, s)$.

Although the operators $A_{\lambda}$ satisfy the more complicated product rule

$$
A_{\mu} A_{\nu}=\sum_{\lambda} m\left[\begin{array}{c}
\lambda \\
\mu
\end{array}\right] A_{\lambda},
$$

it is still true that the operators $A_{n}$ corresponding to one part partitions generate the others. Let us show, for example, how to express the operators corresponding to two part partitions in terms of the operators $A_{n}$. There are two cases. First let $\lambda=(m, n), m \neq n$; then, since $A_{m} A_{n}=A_{\lambda}+A_{m+n}$, we have $A_{\lambda}=$ $A_{m} A_{n}-A_{m+n}$. On the other hand if $\lambda=\left(n^{2}\right), A_{n}^{2}=2 A_{\lambda}+A_{2 n}$; so that $A_{\lambda}=\left(A_{n}^{2}-A_{2 n}\right) / 2$. Similarly operators belonging to three part partitions may be expressed in terms of the operators belonging to one and two part partitions, and so forth. In this sense all the operators $A_{\lambda}$ are determined by the operators $A_{n} . A_{n}$ is a derivation, hence is completely described by

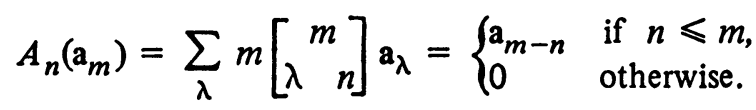

The operator $A_{n}$ corresponds to the operator $d_{n}$ in MacMahon [9]. 
The complete homogeneous symmetric functions. The polynomial $\mathrm{h}(A, \lambda)$ counts all tableaux of shape $\lambda$ and entries from $A$. h satisfies the identities

$$
\begin{gathered}
\mathrm{h}(A \oplus B, \lambda)=\sum_{\mu, \nu} m\left[\begin{array}{l}
\lambda \\
\mu
\end{array}\right] \mathrm{h}(A,) \mathrm{h}(B,), \\
\mathbf{h}(A, \mu) \mathrm{h}(A, \nu)=\mathbf{h}(A, \mu \oplus \nu) .
\end{gathered}
$$

Thus $\mathbf{h}$ is a morph from $\mathbf{N}$ to $(\mathrm{Pn}, m, s)$. Concerning the operators $H_{\lambda}$ we have

$$
H_{\mu} H_{\nu}=\sum_{\lambda} m\left[\begin{array}{cc}
\lambda \\
\mu & \nu
\end{array}\right] H_{\lambda}
$$

The operators $H_{n}$ are derivations and

$$
H_{n}\left(h_{m}\right)= \begin{cases}h_{m-n} & \text { if } n \leqslant m, \\ 0 & \text { otherwise. }\end{cases}
$$

The Schur functions. These are the most interesting of all symmetric functions, but from the point of view of binomial enumeration their theory is in an unsatisfactory state. The polynomial $\mathrm{e}(A, \lambda)$ counts column strict tableaux, that is tableaux whose columns are strictly increasing when read down. Even the symmetry of $\mathrm{e}(A, \lambda)$ is difficult to prove directly from this combinatorial definition (see [16]). Littlewood [8, Chapter VI] has proven that e satisfies the identities,

$$
\begin{gathered}
\mathrm{e}(A \oplus B, \lambda)=\sum_{\mu, \nu} g\left[\begin{array}{ll}
\lambda \\
\mu & \nu
\end{array}\right] \mathrm{e}(A, \mu) \mathrm{e}(B, \nu), \\
\mathrm{e}(A, \mu) \mathrm{e}(B, \nu)=\sum_{\lambda} g\left[\begin{array}{cc}
\lambda \\
\mu
\end{array}\right] \mathrm{e}(A, \lambda),
\end{gathered}
$$

where the incidence coefficients $g$ count a special class of column strict tableaux. Thus $\mathrm{e}$ is a morph from $\mathbf{N}$ to $(\mathrm{Pn}, \mathrm{g}, \mathrm{g})$. The proof of (16) and (17) given by Littlewood involves many properties of the character table of the symmetric groups. We shall not develop these here. The differential operators $E_{\lambda}$ were first defined and applied by Foulkes [4].

7. Connection constants. The table below summarizes some facts concerning the connection constants among the four basic classes of symmetric functions within our present view. Following $\$ 4$ each set is itself a morphism. These constants are of considerable combinatorial interest in their own right, but, in addition, it is through their study that we are able to prove identities relating the different classes of symmetric functions. 


\begin{tabular}{|c|c|c|c|}
\hline Symbol & Definition & Morphism & Combinatorial Interpretation \\
\hline $\mathbf{m}(\mu, \lambda)$ & $h_{\lambda}=\Sigma m(\mu, \lambda) k_{\mu}$ & $(\mathrm{Pn}, s, m) \rightarrow(\mathrm{Pn}, m, s)$ & $\begin{array}{l}\text { \#matrices with row sums } \mu \\
\text { and column sums } \lambda\end{array}$ \\
\hline$\overline{\mathbf{m}}(\mu, \lambda)$ & $\mathbf{a}_{\lambda}=\Sigma \overline{\mathbf{m}}(\mu, \lambda) \mathbf{k}_{\mu}$ & $(\mathrm{Pn}, s, m) \rightarrow(\mathrm{Pn}, m, s)$ & $\begin{array}{l}\text { \#matrices of zeros and ones with } \\
\text { row sums } \mu \text {, column sums } \lambda\end{array}$ \\
\hline $\mathbf{r}(\mu, \lambda)$ & $\mathbf{s}_{\lambda}=\Sigma \quad \mathbf{r}(\mu, \lambda) \mathbf{k}_{\mu}$ & $(\mathrm{Pn}, s, m) \rightarrow(\mathrm{Pn}, b, s)$ & $\begin{array}{l}\text { \#matrices with one nonzero entry } \\
\text { per row, row sums } \mu \text {, column } \\
\text { sums } \lambda\end{array}$ \\
\hline $\mathbf{p}(\mu, \lambda)$ & $\mathbf{h}_{\lambda}=\Sigma \mathbf{p}(\mu, \lambda) \mathbf{s}_{\mu}$ & $(\mathrm{Pn}, b, s) \rightarrow(\mathrm{Pn}, m, s)$ & $\begin{array}{l}\text { cycle index of the symmetric } \\
\text { group } S_{\lambda}=S_{\lambda_{1}} \oplus S_{\lambda_{2}} \oplus \cdots\end{array}$ \\
\hline$\overline{\mathbf{p}}(\mu, \lambda)$ & $\mathbf{a}_{\lambda}=\Sigma \overline{\mathbf{p}}(\mu, \lambda) \mathrm{s}_{\mu}$ & $(\mathrm{Pn}, b, s) \rightarrow(\mathrm{Pn}, m, s)$ & $\operatorname{sgn}(\mu) p(\mu, \lambda)$ \\
\hline $\mathbf{t}(\mu, \lambda)$ & $a_{\lambda}=\Sigma t(\mu, \lambda) h_{\mu}$ & $(\mathrm{Pn}, m, s) \rightarrow(\mathrm{Pn}, m, s)$ & $\begin{array}{l}\operatorname{sgn}(\mu)(\# \text { compositions of the multi- } \\
\text { partite number } \lambda \text { using parts } \mu \text { ) }\end{array}$ \\
\hline
\end{tabular}

All our results concerning symmetric polynomials follow from the simple relations

$$
\begin{gathered}
\mathrm{s}_{n}=\mathrm{k}_{n}, \\
\mathrm{~h}_{n}=\sum_{\|\lambda\|=n} \mathrm{k}_{\lambda}, \\
\mathrm{a}_{n}=\mathrm{k}_{1} n .
\end{gathered}
$$

Let us begin by verifying the combinatorial interpretations of $m, \bar{m}$, and $r$. We have

$$
\begin{aligned}
\mathrm{m}(\mu, \lambda) & =\left\langle K_{\mu}, \mathbf{h}_{\lambda}\right\rangle=\left\langle K_{\mu}, \mathbf{h}_{\lambda_{1}} \mathbf{h}_{\lambda_{2}} \cdots\right\rangle \\
& =\sum_{\nu_{1}, \nu_{2}, \cdots} m\left[\begin{array}{cc}
\mu \\
\nu_{1} & \nu_{2}
\end{array}\right] .\left\langle K_{\nu_{1}}, \mathbf{h}_{\lambda_{1}}\right\rangle\left\langle K_{\nu_{2}}, \mathbf{h}_{\lambda_{2}}\right\rangle \cdots,
\end{aligned}
$$

where $\lambda=\left(\lambda_{1}, \lambda_{2}, \cdots\right)$, and we have used the Leibnitz formula for $K_{\mu}$ applied to a product. This $m\left[\nu_{1}, \nu_{2} \ldots\right]$ is the $m$ multinomial coefficient and counts the number of matrices with $|\mu|$ rows and $|\lambda|$ columns whose row sums are the parts of $\mu$, while the nonzero elements of each column are the parts of the corresponding $v_{i}$. Continuing,

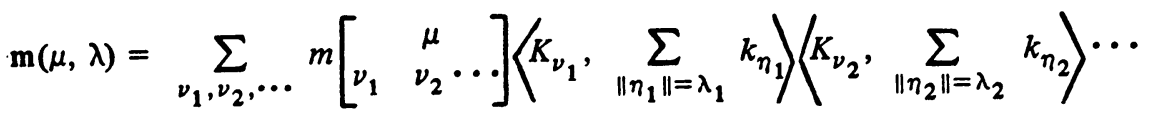

$$
\begin{aligned}
& =\sum_{\left\|\nu_{1}\right\|=\lambda_{1} ;\left\|\nu_{2}\right\|=\lambda_{2} ; \cdots} m\left[\begin{array}{cc}
\mu \\
\nu_{1} & \nu_{2} \cdots
\end{array}\right] \text {. }
\end{aligned}
$$

In view of the combinatorial interpretation of the incidence coefficients $m$, this is the desired conclusion. Analogous arguments apply to $\overline{\mathbf{m}}$ and $\mathbf{r}$. 
It is clear that $\mathrm{m}$ is symmetric, $\mathrm{m}(\mu, \lambda)=\mathrm{m}(\lambda, \mu)$. To exploit this, following MacMahon, consider the operator $M$ from $P$ to $P^{*}$ defined by setting $M\left(\mathrm{k}_{\lambda}\right)=H_{\lambda}$, and extending by linearity. Since the operators $H_{\lambda}$ and the symmetric functions $\mathbf{k}_{\lambda}$ satisfy the same multiplication law, $M$ is an algebra homomorphism. Now by $\S 4$, we find $M\left(\mathrm{~h}_{\lambda}\right)=\Sigma_{\mu} \mathrm{m}(\mu, \lambda) H_{\mu}=\Sigma_{\mu} \mathrm{m}(\lambda, \mu) H_{\mu}=$ $K_{\lambda}$. It follows that to every identity among the functions $\mathbf{h}_{\lambda}, \mathbf{k}_{\lambda}$ there corresponds the same identity among the operators $K_{\lambda}, H_{\lambda}$ and vice versa with the letters ' $h$ ' and ' $k$ ' interchanged. The same analysis applies to $\overline{\mathbf{m}}$, so there is a similar homomorphism $\bar{M}: \mathrm{P} \rightarrow \mathrm{P}^{*}$ with $\bar{M}\left(\mathrm{a}_{\lambda}\right)=K_{\lambda}$ and $\bar{M}\left(\mathrm{k}_{\lambda}\right)=A_{\lambda}$.

From (2) and (3) we thus deduce

$$
\begin{gathered}
K_{n}=\sum_{\|\lambda\|=n} H_{\lambda}, \\
K_{n}=A_{1}{ }^{n} .
\end{gathered}
$$

Using (6.12) we find $K_{12}=K_{1}^{2}=A_{1}^{2}=2 A_{12}+A_{2}=2 K_{2}+A_{2}$ or $\mathrm{k}_{2}=$ $\mathbf{a}_{12}-2 \mathbf{a}_{2}$. Continuing in this way one may systematically invert $\overline{\mathbf{m}}$. In another direction, following MacMahon,

$$
\begin{aligned}
& A_{n} K_{m}=A_{n} A_{1} m=A_{n+1,1} m-1+A_{n, 1} m, n>1, \\
& A_{1} K_{m}=A_{1} A_{1} m=A_{2,1} m-1+(m+1) A_{1} m+1
\end{aligned}
$$

Therefore

$$
A_{n}-A_{n-1} K_{1}+A_{n-2} K_{2}-\cdots+(-1)^{n-1} A_{1} K_{n-1}=(-1)^{n-1} n K_{n},
$$

or

$$
(-1)^{n-1} n a_{n}=k_{n}-k_{n-1} a_{1}+\cdots+(-1)^{n-1} k_{1} a_{n-1} .
$$

It is not necessary to manipulate operators in this fashion to find identities. Any identity involving $a_{\lambda}, h_{\lambda}$ and $k_{\lambda}$ may be proven by expanding with respect to one or another of these bases. For example let us find an expression in terms of $k_{\lambda}$ for the nonalternating sum $k_{n}+k_{n-1} a_{1}+\cdots+k_{1} a_{n-1}$. We find that

$$
\begin{aligned}
\left\langle K_{\lambda}, \sum_{0}^{n-1} a_{k} k_{n-k}\right\rangle=\sum_{0}^{n-1} \sum_{\mu, \nu} m\left[\begin{array}{c}
\lambda \\
\mu
\end{array}\right]\left\langle K_{\mu}, k_{1}\right\rangle\left\langle K_{\nu}, k_{n-k}\right\rangle \\
=\sum_{0}^{n-1} m\left[\begin{array}{cc}
\lambda \\
1^{k} & n-k
\end{array}\right]= \begin{cases}0 & \text { if } \lambda \neq\left(1^{k}, n-k\right), 0 \leqslant k \leqslant n, \\
2 & \text { if } \lambda=\left(1^{k}, n-k\right), k \leqslant n-2, \\
n & \text { if } \lambda=\left(1^{n}\right) ;\end{cases}
\end{aligned}
$$


therefore

$$
k_{n}+k_{n-1} a_{1}+\cdots+k_{1} a_{n-1}-n a_{n}=2\left(k_{n}+k_{1, n-1}+\cdots+k_{1 n-2,2}\right) \text {. }
$$

For later use we state the analogous result for $h$ :

$$
k_{n}+k_{n-1} h_{1}+\cdots+k_{1} h_{n-1}=n h_{n} \text {. }
$$

We can use $M$ to define an inner product on $P$,

$$
[f, g]=\langle M f, g\rangle, \quad f, g \in P .
$$

This amounts to setting $\left[h_{\mu}, k_{\nu}\right]=\delta_{\mu, \nu}$. This inner product is symmetric as a consequence of the symmetry of $\mathbf{m}$.

The connection constants $\mathbf{r}$, while not symmetric, possess an important triangular property. Let $\lambda, \mu$ be partitions. We say $\lambda$ dominates $\mu$, written $\mu \ll \lambda$, whenever the parts of $\lambda$ when added together in suitable groups yield the parts of $\mu$. (When the order of the parts within each group is considered important, this is called a composition of $\mu$ using the parts of $\lambda$.) From its combinatorial interpretation, we deduce that $\mathbf{r}(\mu, \lambda)$ is zero unless $\mu \ll \lambda$.

Passing now to connections among $s_{\lambda}, h_{\lambda}$, and $a_{\lambda}$, we state the fundamental result

$$
n S_{n}=H_{n}=(-1)^{n+1} A_{n}
$$

Since $S_{n}=\Sigma_{\lambda} \mathrm{p}(n, \lambda) H_{\lambda}=\Sigma_{\lambda} \overline{\mathrm{p}}(n, \lambda) A_{\lambda}$, this is equivalent to

(7.10) $\mathbf{p}(n, \lambda)=\left\{\begin{array}{ll}0, & \lambda \neq n, \\ 1 / n, & \lambda=n,\end{array} \quad \overline{\mathbf{p}}(n, \lambda)= \begin{cases}0 & \lambda \neq n, \\ (-1)^{n+1} / n, & \lambda=n .\end{cases}\right.$

To prove (10) we first note that as $s_{\lambda}, a_{\lambda}$ and $h_{\lambda}$ are homogeneous of degree $\|\lambda\|, \mathbf{p}(n, \lambda)$ is zero unless $n=\|\lambda\|$. Using the morphism property of $\mathbf{p}$, if $\lambda$ is not a one part partition, then $\lambda$ has a nontrivial separation $\lambda=\lambda_{1} \oplus \lambda_{2}$, with $\left|\lambda_{1}\right|,\left|\lambda_{2}\right|<n$, and $\mathbf{p}(n, \lambda)=\mathrm{p}\left(n, \lambda_{1}\right)+\mathrm{p}\left(n, \lambda_{2}\right)=0$. The same argument applies to $\overline{\mathrm{p}}$. To conclude, we must verify that $\mathrm{p}(n, n)=1 / n$. From (7),

$$
h_{n}=s_{n} / n+n^{-1}\left(s_{n-1} h_{1}+\cdots+s_{1} h_{n-1}\right) \text {, }
$$

from which this conclusion follows, since the second term when expanded in terms of $s$ can only contain terms involving $s_{\lambda}$, where $|\lambda|>1$ (see (6.5)). The same argument applied to (6) concludes the proof of (10).

As an application of (9) we find a closed form for $\mathrm{p}(\mu, \lambda), \mu=\left(\mu_{1} \mu_{2}\right.$ 


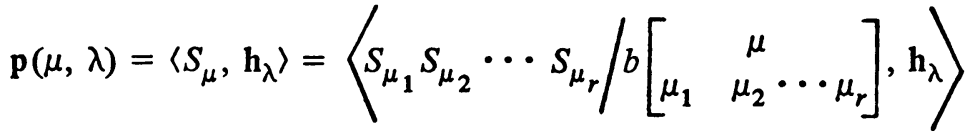

$$
\begin{aligned}
& =\frac{1}{\mu !}\left\langle\frac{H_{\mu_{1}}}{\mu_{1}} \frac{H_{\mu_{2}}}{\mu_{2}} \cdots \frac{H_{\mu_{r}}}{\mu_{r}}, \mathrm{~h}_{\lambda}\right\rangle
\end{aligned}
$$

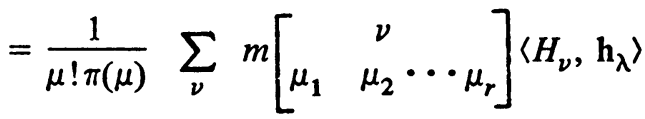

$$
\begin{aligned}
& =m\left[\begin{array}{cc} 
& \lambda \\
\mu_{1} & \mu_{2} \\
\cdots & \mu_{r}
\end{array}\right] / \mu ! \pi(\mu) .
\end{aligned}
$$

In particular, $\mathbf{p}(\mu, n)=1 / \mu ! \pi(\mu)$ when $\|\mu\|=n$, thus

$$
\mathbf{h}_{n}=\sum_{\|\mu\|=n} \frac{\mathbf{s}_{\mu}}{\mu ! \pi(\mu)} .
$$

The differential operators provide in principle a tool for proving or disproving any identity in the symmetric functions. We can expand both sides of any proposed identity with respect to a convenient basis and check corresponding coefficients. Such an expansion is always possible, for by using the product rules and Leibnitz rules, such an expansion is reduced to the evaluation of terms of the form $\left\langle B_{\mu}\right.$, $\mathbf{c}_{\lambda}$, where $B$ is $K, H, A$ or $S$, and $\mathbf{c}$ is $\mathbf{k}, \mathbf{h}, \mathbf{a}$, or $\mathbf{s}$. These in turn can be reduced using (4), (5) and (9) to terms of the form $\left\langle B_{\mu}, b_{\lambda}\right\rangle=\delta_{\mu, \lambda}$. The final expressions for the coefficients will involve summations and products of the incidence coefficients $m$. In practice, therefore, this device is limited by our knowledge of these incidence coefficients.

We conclude this section by verifying the combinatorial interpretations of the coefficients $\mathbf{p}$ and $\overline{\mathbf{p}}$. Both of these are morphisms $(\mathbf{P n}, s) \rightarrow(\mathbf{P n}, s)$; that is they satisfy the identity (here stated for $\mathbf{p}$ )

$$
\mathrm{p}\left(\mu, \lambda_{1} \oplus \lambda_{2}\right)=\sum_{\mu=\mu_{1} \oplus \mu_{2}} \mathrm{p}\left(\mu_{1}, \lambda_{1}\right) \mathrm{p}\left(\mu_{2}, \lambda_{2}\right) .
$$

In addition, the cycle index of the symmetric groups $S_{\lambda}$ satisfy the same identity (equation (2.8)). Thus it suffices to verify the equality of $\mathrm{p}(\mu, n)$ with the cycle index of $S_{n}$. This in turn follows directly from equation (11). We can now complete the formal derivation of Pólya's enumeration theorem begun in $\S 3$. From our current standpoint, we see that the connection constants between the shifts $E_{\lambda}$ of $\S 3$, Example 5 and the differential operators $P_{\mu}$ are just $\mathrm{p}(\mu, \lambda)$. These were inverted in $\S 3$ in order to express $P_{\mu}$ in terms of $E_{\lambda}$. Let $q$ stand 
for the inverse of p. Then $P_{\lambda}=\Sigma_{\mu} q(\mu, \lambda) E_{\mu}$, and also $s_{\lambda}=\Sigma_{\mu} q(\mu, \lambda) h_{\lambda}$. We must verify $\left\langle P_{\lambda}, f\right\rangle=\Sigma_{\mu} \mathrm{q}(\mu, \lambda)\left\langle E_{\mu}, f\right\rangle=\mathrm{s}_{\lambda}$ (compare with equation (3.10)). This now follows directly from $\left\langle E_{\mu}, f\right\rangle=f\left(S_{\mu}\right)=h_{\mu}$, which in turn is a more or less direct consequence of the definition of $f$.

8. An umbral operator. Among all the operators on $P$ represented by the connection constants of our table, only one is an umbral operator. This is the operator $U$ defined by $U h_{\lambda}=a_{\lambda}$, whose matrix with respect to the $h_{\lambda}$ is $t$. The operator $U$ is umbral because $U$ maps $h$ to a morph a which uses the same incidence coefficients (namely $m$ ) as h. It follows that $U$ applied to any morph yields a morph which uses the same incidence coefficients.

Consider first $U$ applied to $s_{\lambda}$. The result will yield another morph from $\mathrm{N}$ to $(\mathrm{Pn}, b, s)$ with the associated differential operators $U S_{\lambda} U^{-1}$. By (7.9), $U S_{n} U^{-1}=(-1)^{n+1} S_{n}$. Therefore, by (6.6), $U S_{\lambda} U^{-1}=\operatorname{sgn}(\lambda) S_{\lambda}$. Thus the matrix of connection constants, which by $\S 3$ link $S_{\lambda}$ and $U S_{\lambda} U^{-1}$, is diagonal with diagonal elements $\operatorname{sgn}(\lambda)$. The inverse matrix is clearly the same matrix, thus we conclude that $U$ is its own inverse and

$$
U s_{\lambda}=\operatorname{sgn}(\lambda) s_{\lambda} .
$$

The functions $s_{\lambda}$ are then a complete set of eigenvectors for $U$. Since $U$ exchanges two multiplicative bases, $U$ is a homomorphism. It follows that in every identity involving $a_{\lambda}$ and $h_{\lambda}$ we may exchange the two letters. In the terminology of $\S 3, a_{\lambda}$ and $h_{\lambda}$ are each self-inverse with respect to the other.

An important property of the $s_{\lambda}$ in this connection is their orthogonality with respect to the inner product (6.8). This is established as follows.

$$
\left[\mathbf{s}_{\mu}, \mathbf{s}_{\lambda}\right]=\left[\sum_{\nu} \mathbf{q}(\nu, \mu) \mathbf{h}_{\nu}, \sum_{\eta} \mathbf{r}(\eta, \lambda) \mathbf{k}_{\eta}\right]=\sum_{\nu} \mathbf{q}(\nu, \mu) \mathbf{r}(\nu, \lambda) .
$$

But $\mathbf{q}(\nu, \mu)$ is zero unless $\nu \gg \mu$ while $\mathbf{r}(\nu, \lambda)$ is zero unless $\nu \ll \lambda$; therefore if $\mu \gg \lambda$ or if $\mu$ and $\lambda$ are incomparable, $\left[s_{\mu}, s_{\lambda}\right]=0$. Thus the $s_{\lambda}$ form an orthogonal basis with $\left[\mathbf{s}_{\lambda}, \mathbf{s}_{\lambda}\right]=q(\lambda, \lambda) \mathbf{r}(\lambda, \lambda)$. Using (2.9) and the combinatorial interpretation of $\mathbf{r}$, we find that $q(\lambda, \lambda)=\pi(\lambda), \mathbf{r}(\lambda, \lambda)=\lambda$ !. Thus $\left[s_{\lambda}, s_{\lambda}\right]=\pi(\lambda) \lambda$ !. It follows that the inner product is positive definite, and $U$ is an isometry.

Consider now the images of $\mathbf{k}_{\lambda}$ under $U$. Setting $\mathbf{f}_{\lambda}=U \mathbf{k}_{\lambda}$ we obtain a morph from $\mathbf{N}$ to $(\mathbf{P n}, s, m)$. The connections between this new set of symmetric functions and the others are summarized by the relations $F_{n}=H_{1 n}=$ $\Sigma_{\|\lambda\|={ }_{n}} A_{\lambda}$, which follow immediately from (7.4) and (7.5). We also have $\mathbf{f}_{n}=$ $U\left(\mathbf{k}_{n}\right)=U\left(s_{n}\right)=(-1)^{n+1} \mathbf{k}_{n}$. The morphs $\mathbf{f}$ and $\mathbf{k}$ are each self-inverse with respect to the other, and any identity involving them both remains an identity upon 
exchanging letters. Also since $M\left(\mathrm{f}_{n}\right)=(-1)^{n+1} M\left(\mathrm{k}_{n}\right)=(-1)^{n+1} H_{n}=A_{n}$, we must have $M\left(\mathrm{f}_{\lambda}\right)=A_{\lambda}$ and $M\left(\mathrm{a}_{\lambda}\right)=F_{\lambda}$. The functions $\mathrm{f}_{n}$ were first investigated by Doubilet [3].

Many of these connections among symmetric functions are summarized in the properties of the symmetric functions $d_{\mu, \lambda}(\mu, \lambda \in P n)$ defined by

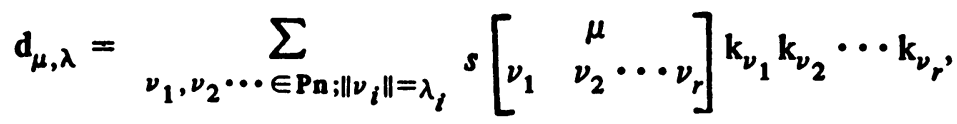

where $\lambda=\left(\lambda_{1}, \lambda_{2}, \cdots, \lambda_{r}\right)$. These were first discussed by MacMahon [9]. Among the functions $d_{\mu, \lambda}$ appear all the other classes of symmetric functions, thus $d_{1, n}=a_{\lambda}, d_{\mu, n}=k_{\mu}, d_{\lambda, \lambda}=s_{\lambda}, \Sigma_{\mu} d_{\mu, \lambda}=h_{\lambda}$. Note that $d_{\mu, \lambda}$ is zero unless $\mu \gg \lambda$. Expanding with respect to $k$, we get $d_{\mu, \lambda}=\Sigma_{\nu \mu} \theta_{\lambda, \nu} k_{\nu}$, where

$$
\mu^{\theta} \theta_{\lambda, \nu}=\sum_{\nu_{1}, \nu_{2}, \cdots, \nu_{r}} s\left[\begin{array}{c}
\mu \\
\nu_{1} \cdots \nu_{r}
\end{array}\right] m\left[\begin{array}{c}
\nu \\
\nu_{1} \cdots \nu_{r}
\end{array}\right] .
$$

The coefficient ${ }_{\mu} \theta_{\lambda, \nu}$ counts the number of matrices with row sums $\nu$ and column sums $\lambda$ whose nonzero entries are the parts of $\mu$. These coefficients have a symmetry property $\left({ }_{\mu} \theta_{\lambda, \nu}={ }_{\mu} \theta_{\nu, \lambda}\right)$ and a diagonal property $\left({ }_{\mu} \theta_{\lambda, \nu}=0\right.$ unless $\mu \gg \lambda, \nu$ ).

From our point of view the most important property of $d_{\mu, \lambda}$ is given by the following identity:

$$
d_{\mu, \lambda_{1} \oplus \lambda_{2}}=\sum_{\mu=\mu_{1} \oplus \mu_{2}} d_{\mu_{1}, \lambda_{1}} d_{\mu_{2}, \lambda_{2}} .
$$

Thus $\mathrm{d}$ is a morphism of $(\mathbf{P n}, s) \leftarrow(\mathbf{P n}, s)$ (with values in $P$ rather than real values). The proof of (2), as the reader will easily verify, depends only on the property

$$
s\left[\begin{array}{cc}
\mu \\
\nu_{1} \cdots \nu_{r} & \gamma_{1} \cdots \gamma_{s}
\end{array}\right]=\sum_{\mu_{1}, \mu_{2}} s\left[\begin{array}{c}
\mu \\
\mu_{1}
\end{array}\right] s\left[\begin{array}{c}
\mu_{1} \\
\nu_{1} \cdots \nu_{r}
\end{array}\right] s\left[\begin{array}{c}
\mu_{2} \\
\gamma_{1} \cdots \gamma_{s}
\end{array}\right]
$$

of the multinomial coefficients $s$, not on any property of the symmetric functions $k$.

The same identity (2) is satisfied by all connection constants between multiplicative bases (see (7.12)). The values of these connection constants, as we have argued earlier, are determined by their values when $\lambda=n=\|\mu\|$. In the case of $\mathbf{d}$ these are $\mathbf{d}_{\mu, n}=\mathbf{k}_{\mu}$. If $Q$ is a shift invariant operator on $P$, then setting $\mathbf{q}(\mu, \lambda)=\left\langle Q, \mathbf{d}_{\mu, \lambda}\right\rangle$ defines a real-valued morphism $(\mathrm{Pn}, s) \leftarrow(\mathrm{Pn}, s)$, provided $Q$ is a homomorphism of $P$. This follows by applying $Q$ to (2) and 
taking inner products. If we wish to represent a specific $\mathbf{q}$ in this fashion; the operator $Q$, which we may term the umbral representation of $q$, is determined by $\left\langle Q, \mathrm{k}_{\mu}\right\rangle=\mathrm{q}(\mu, n)$. The condition that $Q$ be a homomorphism turns out to be equivalent to $q$ being a morphism from $(P n, m)$ to $(P n, m)$.

Consider, in particular, the shift invariant operator $T$ defined in this way by the connection constants $\mathrm{t}, T=\Sigma_{\mu} \mathrm{t}(\mu, n) K_{\mu}, n=\|\mu\|$. A closed form for $T$ may be found as follows,

$$
M^{-1}(T)=\sum_{\mu} \mathrm{t}(\mu, n) M^{-1}\left(K_{\mu}\right)=\sum_{\mu} \mathrm{t}(\mu, n) \mathrm{h}_{\mu}=\sum_{n} \mathrm{a}_{n} .
$$

Hence $T=M\left(\Sigma_{n} a_{n}\right)=\Sigma_{n} F_{n}$. We now establish the important identity

$$
K_{n}-K_{n-1} F_{1}+\cdots+(-1)^{n} F_{n}= \begin{cases}I & \text { if } n=0, \\ 0 & \text { if } n>0 .\end{cases}
$$

This follows from computing

$$
\begin{aligned}
F_{k} K_{n-k} & =H_{1}\left(\sum_{\|\mu\|=n-k} H_{\mu}\right) \\
& =\sum_{\|\nu\|=n ;\|\mu\|=n-k} m\left[\begin{array}{cc}
\nu \\
1^{k} & \mu
\end{array}\right] H_{\nu}=\sum_{\|\nu\|=n}\left(\begin{array}{c}
|\nu| \\
k
\end{array}\right) H_{\nu}
\end{aligned}
$$

so that

$$
\sum_{k=0}^{n}(-1)^{k} F_{k} K_{n-k}=\sum_{\|\nu\|=n} H_{\nu} \sum_{k}(-1)^{k}\left(\begin{array}{l}
|\nu| \\
k
\end{array}\right)= \begin{cases}I, & n=0, \\
0, & n<0 .\end{cases}
$$

From (3) we obtain the familiar identity $h_{n}-h_{n-1} a_{1}+\cdots+(-1)^{n} a_{n}=\delta_{0, n}$ by applying $M$. More important in the present context, we have from (3),

$$
\begin{aligned}
T & =\left(I+F_{1}+F_{2}+\cdots\right)=I /\left(I-K_{1}+K_{2}-\cdots\right) \\
& =I+\left(K_{1}-K_{2}+\cdots\right)+\left(K_{1}-K_{2}+\cdots\right)^{2}+\cdots \\
& =\sum_{\mu} \operatorname{sgn}(\mu) b(\mu) K_{\mu} .
\end{aligned}
$$

Thus $\mathbf{t}(\mu, n)=\operatorname{sgn}(\mu) b(\mu), n=\|\mu\|$.

9. Two problems. The Schur functions possess the most interesting connection constants, for $s_{\lambda}=\Sigma_{\mu} \chi_{\lambda}^{(\mu)} e_{\mu}$, where $\chi_{\lambda}^{(\mu)}$ is the character table of the symmetric groups. It would, therefore, be very interesting to find a purely combinatorial proof of (6.16) and (6.17). Then the techniques of $\S \S 7$ and 8 could be used to derive theorems about the characters. For example, the Schur functions also turn out to be an orthogonal basis for $P$, and this implies the usual 
orthogonality relations for $\chi_{\lambda}^{(\mu)}$. The same ideas would almost certainly lead to a parallel development of the Hall polynomials (see [6]) leading in turn to the character theory of the finite general linear groups. The connection constants between the Hall polynomials and $s_{\lambda}$ are essentially the polynomials $Q_{q}(\lambda, p)$ defined by Green and proven by him to satisfy an identity of binomial type [6, p. 420].

We must mention also the work of Andrews [1], who has developed a theory for the solutions of the identity

$$
p_{n}(x+y)=\sum_{k}\left(\begin{array}{l}
n \\
k
\end{array}\right)_{q} q^{x(n-k)} p_{k}(x) p_{n-k}(y),
$$

where $p_{n}$ is a polynomial of degree $n$ in $q^{x}$ and $\left(\begin{array}{c}n \\ k\end{array}\right)_{q}$ are the Gaussian coefficients. Our theory does not apply due to the appearance of $x$ in the incidence coefficient $\left(\begin{array}{c}n \\ k\end{array}\right)_{q} q^{x(n-k)}$. The generalization of Andrews' result in the direction of this paper would have a flavor analogous to the theory of projective representations in the theory of group representations. Such a generalization would apply not only to polynomial sequences satisfying (1), but also to Stanley's generating functions $W(s, P)(s \in \mathbf{Z}, P \in \mathbf{P})$ [15, p. 23 and following, especially p. 38], and the Ore polynomials [11] which we conjecture satisfy a similar identity.

\section{Index of Principal Identities}

Identity
(A) associativity
(A') associativity for the second pair of incidence coefficients
(B) binomial identity
(B') double morph definition
(D) definition of differential operators
(D') dual action of $P$ on $P^{*}$

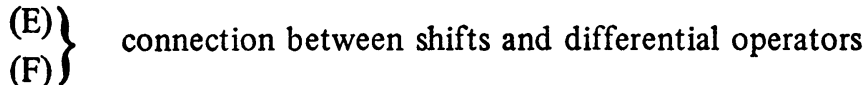
$\left(\mathrm{F}^{\prime}\right)$ connection between the dual action and multiplication operators
(G) connection of shifts and inner product
9, 20
(H) Hopf identity
(I) inverse identity
$\left.\begin{array}{l}\text { (L) } \\ \left(L^{\prime}\right)\end{array}\right\}$ Leibnitz rules
(M) multiplication rule for differential operators 8
$\begin{array}{ll}\left(M^{\prime}\right) \text { multiplication rule for } \mathbf{p} & 19\end{array}$
(N) negative shift identity
(P) product rule for shifts
(S) definition of shifts 


\section{REFERENCES}

1. G. Andrews, On the foundations of combinatorial theory. V: Eulerial differential operators, Studies in Appl. Math. 50 (1971), 345-375.

2. T. Brylawski, $A$ decomposition for combinatorial geometries, Trans. Amer. Math. Soc. 171 (1972), 235-282. MR 46 \#8869.

3. P. Doubilet, On the foundations of combinatorial theory. VII: Symmetric functions through the theory of distribution and occupancy (preprint).

4. H. O. Foulkes, Differential operators associated with S-functions, J. London Math. Soc. 24 (1949), 136-143. MR 11, 4.

5. A. Garsia, An expose of the Mullin-Rota theory of polynomials of binomial type, J. Multilinear Algebra (1973).

6. J. A. Green, The characters of the finite general linear groups, Trans. Amer. Math. Soc. 80 (1955), 402-447. MR 17, 345.

7. M. Henle, Dissection of generating functions, Studies in Appl. Math. 51 (1972), $397-410$.

8. D. E. Littlewood, The theory of group characters and matrix representations of groups, Oxford Univ. Press, Oxford, 1940. MR 2, 3.

9. P. MacMahon, Combinatory analysis, Chelsea, New York, 1960. MR 25 \#5003.

10. R. Mullin and G.-C. Rota, On the foundations of combinatorial theory, III: Theory of binomial enumeration, Graph Theory and its Applications (Proc. Advanced Sem., Math. Research Center, Univ. of Wisconsin, Madison, Wis., 1969), Academic Press, New York, 1970, pp. 107-213. MR 43 \#65.

11. O. Ore, On a special class of polynomials, Trans. Amer. Math. Soc. 35 (1933) 559-584.

12. R. C. Read, The use of $S$-functions in combinatorial analysis, Canad. J. Math. 20 (1968), 808-841. MR 37 \#5108.

13. J. Riordan, An introduction to combinatorial analysis, Wiley Publ. in Math. Statist., Wiley, New York, 1958. MR 20 \#3077.

14. G.-C. Rota, On the foundations of combinatorial theory. VIII: Finite operator calculus, J. Math. Anal. Appl. 42 (1973).

15. R. Stanley, Ordered structures and partitions, Mem. Amer. Math. Soc. No. 119 (1972).

16. - Theory and applications of plane partitions. I, Studies in Appl. Math. 50 (1971), 167-188.

17. - A Brylawski decomposition for finite ordered sets, Discrete Math. 4 (1973), 77-82. MR 46 \#8918.

18. P. Doubilet, G.-C. Rota and R. Stanley, On the foundations of combinatorial theory. VI: The idea of generating function, Sixth Berkeley Sympos., Univ. of California Press, Berkeley, Calif., 1972.

DEPARTMENT OF MATHEMATICS, OBERLIN COLLEGE, OBERLIN, OHIO 44074 